\title{
NORMALIZERS AND SELF-NORMALIZING SUBGROUPS
}

\author{
BORIS ŠIROLA \\ University of Zagreb, Croatia
}

\begin{abstract}
Let $\mathbb{K}$ be a field, $\operatorname{char}(\mathbb{K}) \neq 2$. Suppose $G=\boldsymbol{G}(\mathbb{K})$ is the group of $\mathbb{K}$-points of a reductive algebraic $\mathbb{K}$-group $\boldsymbol{G}$. Let $G_{1} \leq G$ be the group of $\mathbb{K}$-points of a reductive subgroup $\boldsymbol{G}_{1} \leq \boldsymbol{G}$. We study the structure of the normalizer $\mathrm{N}=\mathcal{N}_{G}\left(G_{1}\right)$. In particular, let $G=\mathrm{SL}(2 n, \mathbb{K})$ for $n>1$. For certain well known embeddings of $G_{1}$ into $G$, where $G_{1}=\operatorname{Sp}(2 n, \mathbb{K})$ or $\mathrm{SO}(2 n, \mathbb{K})$, we show that $\mathrm{N} / G_{1} \cong \boldsymbol{\mu}_{k}(\mathbb{K})$, the group of $k$-th roots of unity in $\mathbb{K}$. Here, $k=2 n$ if certain Condition $(\diamond)$ holds, and $k=n$ if not. Moreover, there is a precisely defined subgroup $\mathrm{N}^{\prime}$ of $\mathrm{N}$ such that $\mathrm{N} / \mathrm{N}^{\prime} \cong \mathbb{Z} / 2 \mathbb{Z}$ if Condition $(\diamond)$ holds, and $\mathrm{N}=\mathrm{N}^{\prime}$ if not. Furthermore, when $n>1$, as the main observations of the paper we have the following: (i) $\mathrm{N}$ is a self-normalizing subgroup of $G$; (ii) $\mathrm{N}^{\prime} \cong G_{1} \rtimes \boldsymbol{\mu}_{n}(\mathbb{K})$, the semidirect product of $G_{1}$ by $\boldsymbol{\mu}_{n}(\mathbb{K})$. Besides we point out that analogous results will hold for a number of other pairs of groups $\left(G, G_{1}\right)$. We also show that for the pair $\left(\mathfrak{g}, \mathfrak{g}_{1}\right)$, of the corresponding $\mathbb{K}$-Lie algebras, $\mathfrak{g}_{1}$ is self-normalizing in $\mathfrak{g}$; which generalizes a well-known result in the zero characteristic.
\end{abstract}

\section{INTRODUCTION}

Unless specified otherwise, throughout this paper $\mathbb{K}$ is an arbitrary field of characteristic $\neq 2$, and $\overline{\mathbb{K}}$ is its fixed algebraic closure. By $\boldsymbol{\mu}_{n}(\mathbb{K})$ we denote the group of $n$-th roots of unity in $\mathbb{K}$.

An interesting problem in group theory is to study the normalizers $\mathcal{N}_{G}(H)$ for certain subgroups $H$ of a given group $G$. Related to that it is worth searching for some distinguished subgroups $H \leq G$ such that $\mathcal{N}_{G}(H)=H$; i.e., for self-normalizing subgroups of $G$. In support of this claim we should mention here for instance the famous Chevalley normalizer theorem, which

2010 Mathematics Subject Classification. 20E34, 20G15, 17B05, 17B20.

Key words and phrases. Normalizer, self-normalizing subgroup, symmetric pair, symplectic group, even orthogonal group.

The author was supported in part by the Ministry of Science, Education and Sports, Republic of Croatia, Grant No. 037-0372781-2811. 
states that every parabolic subgroup $P$ of a connected affine group $G$ is selfnormalizing, and connected.

The main purpose of this paper is to provide some results concerning the above problem within the class of groups $G=\boldsymbol{G}(\mathbb{K})$ of $\mathbb{K}$-points of reductive algebraic $\mathbb{K}$-groups; $\boldsymbol{G}$ is closed in $\operatorname{GL}(n, \overline{\mathbb{K}})$, for some $n$. More precisely, for such groups $G$ we study some self-normalizing pairs, i.e., pairs $(G, N)$ where $N \leq G$ is self-normalizing. Let us emphasize that our subgroups $N$ will always be the normalizers of certain groups $G_{1}$ that will be the groups of $\mathbb{K}$-points of some reductive or parabolic algebraic $\mathbb{K}$-subgroups $\boldsymbol{G}_{1} \leq \boldsymbol{G}$. Furthermore, the embeddings $G_{1} \hookrightarrow G$ will be useful for various purposes; see Section 4 . For one more observation, suppose for the moment that we restrict ourselves to the simpler situation of algebraic groups. More precisely, let $\mathbb{K}=\overline{\mathbb{K}}$, and moreover $\left(\boldsymbol{G}, \boldsymbol{G}_{1}\right)$ is such that both $\boldsymbol{G}$ and $\boldsymbol{G}_{1}$ are connected. Then the normalizer $\mathcal{N}_{\boldsymbol{G}}\left(\boldsymbol{G}_{1}\right)$ very often will not be connected. This fact makes our consideration more interesting and involved. We should also mention here the seminal work of Dynkin and Seitz who classified the maximal connected subgroups of classical algebraic groups; Dynkin $([\mathrm{Dy} 1, \mathrm{Dy} 2])$ for $\mathbb{K}=\mathbb{C}$, and Seitz $[\mathrm{S}]$ for $\mathbb{K}$ algebraically closed of positive characteristic. We understand our research to be in part complementary to their results. Namely, for connected $\boldsymbol{G}$ and $\boldsymbol{G}_{1}$ as above we will in particular have that the corresponding normalizer is often a maximal (non-connected) subgroup of $\boldsymbol{G}$.

As a precursor for the results we would like to have, it is a good idea first to "translate" our problem into linear algebra. More precisely, one should first try to see what is going on for Lie algebras. Therefore we consider (semisimple) Lie algebras $\mathfrak{g}$ over $\mathbb{K}$, and then we want to understand the set of self-normalizing subalgebras $\mathfrak{g}_{1} \leq \mathfrak{g}$. For that purpose, in Section 2 , we observe that a well known notion of symmetric subalgebra, from the zero characteristic setting, can be straightforwardly generalized in the positive characteristic too. Also, if the pair $\left(\mathfrak{g}, \mathfrak{g}_{1}\right)$, where $\mathfrak{g}_{1} \leq \mathfrak{g}$, is nonsymmetric we can analyze it in a satisfactorily way provided the following condition holds: The restriction of the Killing form of $\mathfrak{g}$, to $\mathfrak{g}_{1}$, is nondegenerate.

Suppose now we have a pair $\left(\mathfrak{g}, \mathfrak{g}_{1}\right)$ consisting of a $\mathbb{K}$-Lie algebra $\mathfrak{g}$ and its subalgebra $\mathfrak{g}_{1}$. If we want to see whether $\mathfrak{g}_{1}$ is self-normalizing in $\mathfrak{g}$, with no loss of generality we can assume that $\mathbb{K}=\overline{\mathbb{K}}$. Namely, suppose that moreover $\mathbb{L} / \mathbb{K}$ is an arbitrary field extension. It is well known that then $N_{\mathfrak{g}}\left(\mathfrak{g}_{1}\right)^{\mathbb{L}} \cong N_{\mathfrak{g}^{\mathbb{L}}}\left(\mathfrak{g}_{1}^{\mathbb{L}}\right)$. As a consequence, we obtain the following equivalence: $\mathfrak{g}_{1}$ is self-normalizing in $\mathfrak{g}$ if and only if $\mathfrak{g}_{1}^{\mathbb{L}}$ is self-normalizing in $\mathfrak{g}^{\mathbb{L}}$. Take now $G=\mathrm{SL}(2, \mathbb{R})$, and consider its subgroup $H_{1}=\left\{g \in G \mid g g^{t}=I\right\}$, the special orthogonal subgroup; see Example 4.7. Then $H_{1}$ is self-normalizing in $G$. On the other hand, for the complexifications $G^{\mathbb{C}}$ and $H_{1}^{\mathbb{C}}$, the quotient $\mathcal{N}_{G^{\mathbb{C}}}\left(H_{1}^{\mathbb{C}}\right) / H_{1}^{\mathbb{C}}$ is isomorphic to $\mathbb{Z} / 2 \mathbb{Z}$; and so $H_{1}^{\mathbb{C}}$ is not self-normalizing in $G^{\mathbb{C}}$. This simple example indicates that while dealing with groups the situation around the problem of self-normalizing subgroups is more complicated. 
In order to explain the present work and in particular formulate our main result, we need a little preparation; see Subsection 3.1. For a matrix $U \in$ $M_{n}(\mathbb{K})$, by $U^{t}$ denote its transpose. Given matrices $X, Y, Z, T \in M_{n}(\mathbb{K})$, define a block-matrix $A=\left(\begin{array}{l}X \\ Z\end{array}\right) \in M_{2 n}(\mathbb{K})$. For $\varepsilon= \pm$, define a map $A \mapsto A_{\varepsilon}^{\sharp}$, where

Then the map

$$
A_{\varepsilon}^{\sharp}=\left(\begin{array}{cc}
T^{t} & \varepsilon Y^{t} \\
\varepsilon Z^{t} & X^{t}
\end{array}\right) .
$$

$$
\theta=\theta^{\varepsilon}: A \mapsto-A_{\varepsilon}^{\sharp}
$$

is an involution on $\mathfrak{G}=\mathfrak{s l}(2 n, \mathbb{K})$; more precisely, $\theta^{-}$(resp. $\left.\theta^{+}\right)$is the symplectic (resp. orthogonal) involution. Let $\mathfrak{G}_{1}^{\varepsilon}=\mathfrak{G}^{\theta}$, the fixed point algebra for $\theta$. Then we have

$$
\mathfrak{G}_{1}^{\varepsilon}=\left\{A \in \mathfrak{G} \mid A_{\varepsilon}^{\sharp}=-A\right\}=\left\{\left(\begin{array}{cc}
X & Y \\
Z & -X^{t}
\end{array}\right) \mid \varepsilon Y^{t}=-Y \text { and } \varepsilon Z^{t}=-Z\right\} \text {. }
$$

Of course, this gives a well known embedding of $\mathfrak{G}_{1}^{-}=\mathfrak{s p}(2 n, \mathbb{K})\left(\right.$ resp. $\mathfrak{G}_{1}^{+}=$ $\mathfrak{s o}(2 n, \mathbb{K}))$ into $\mathfrak{G}$. Next, for $G=\mathrm{SL}(2 n, \mathbb{K})$, define

$$
G_{1}^{\varepsilon}=G_{1}^{ \pm}=\left\{A \in G \mid A A_{\varepsilon}^{\sharp}=I\right\} .
$$

It turns out that $G_{1}^{\varepsilon}$ are subgroups of $G$. More precisely, $G_{1}^{-}$(resp. $G_{1}^{+}$) are realizations of $\operatorname{Sp}(2 n, \mathbb{K})$ (resp. $\operatorname{SO}(2 n, \mathbb{K}))$ within $G$.

Let us now present the content of the paper. Section 1, consisting of two subsections, is preliminary. In the first subsection we fix our notation and conventions. The second subsection, although being quite basic, is crucial for better understanding of what follows. Its purpose is to explain what kind of pairs of groups $\left(G, G_{1}\right)$, and the corresponding pairs of Lie algebras $\left(\mathfrak{g}, \mathfrak{g}_{1}\right)$, we would like to study in general. Our construction of such pairs of groups and Lie algebras, which goes via certain maps $\theta$ (or equivalently, via certain maps $\left.A \mapsto A^{\sharp}\right)$, explains the meaning of the above phrase that " $\left(G, G_{1}\right)$ correspond to $\left(\mathfrak{g}, \mathfrak{g}_{1}\right)$ "; see Proposition 1.3. In Section 2, which might be considered as an appendix to [Š 4 ], we give in brief some general results concerning the pairs $\left(\mathfrak{g}, \mathfrak{g}_{1}\right)$ of Lie algebras we consider, both symmetric and nonsymmetric ones. In Section 3 we prove Theorem 0.1 below, that is our main result. The claim (ii), which is a part of Proposition 3.6, presents the first step toward further generalization of a known result in the case char $(\mathbb{K})=0$; see $[D$, Sect. 1.13], and [Š3] for a generalized result. The claim (i) in particular shows that the structure of the normalizer of $G_{1}$ in $G$ strongly depends on the ground field $\mathbb{K}$; see Theorem 3.7 and Corollary 3.8. Here we would in particular like to emphasize the isomorphism $(0.1)$ below. This, together with the fact that analogous statements we have for a number of other pairs $\left(G, G_{1}\right)$, is the most interesting observation concerning the structure of the normalizer $\mathrm{N}=\mathcal{N}_{G}\left(G_{1}\right)$; cf. the Claim in Example 1.7, and Remark 1.9. Note also that our approach treats simultaneously the symplectic and even orthogonal Lie 
algebras/groups. Some further interesting pairs, and in particular a bit more convoluted case of $\left(G, G_{1}\right)$, when $G_{1}$ is the odd orthogonal group embedded in $G=\mathrm{SL}(2 n+1, \mathbb{K})$, will be treated in [Š5]. Before we give the theorem let us state the following condition on $\mathbb{K}$ and $n$; here, $W^{\sharp}=W_{\varepsilon}^{\sharp}$ is as before.

Condition $(\diamond)$. There exists $\omega \in \boldsymbol{\mu}_{2 n}(\mathbb{K}) \backslash \boldsymbol{\mu}_{n}(\mathbb{K})$ for which we can find some $W \in G$ satisfying $W^{\sharp} W=\omega I$.

THEOREm 0.1. Let $n>1$. Then we have the following:

(i) For $G_{1}=G_{1}\left(\theta^{\varepsilon}\right)$ and the normalizer $\mathrm{N}=\mathcal{N}_{G}\left(G_{1}\right)$, we have

$$
\mathrm{N}=\left\{M \in G \mid M^{\sharp} M=\lambda I, \lambda \in \boldsymbol{\mu}_{2 n}(\mathbb{K})\right\},
$$

and

$$
\mathrm{N} / G_{1} \cong \begin{cases}\boldsymbol{\mu}_{2 n}(\mathbb{K}) & \text { if Condition }(\diamond) \text { holds } \\ \boldsymbol{\mu}_{n}(\mathbb{K}) & \text { otherwise }\end{cases}
$$

More precisely, the set

$$
\mathrm{N}^{\prime}=\left\{M \in G \mid M^{\sharp} M=\lambda I, \lambda \in \boldsymbol{\mu}_{n}(\mathbb{K})\right\}
$$

is a normal subgroup of $\mathrm{N}$, and

$$
\mathrm{N}^{\prime} \cong G_{1} \rtimes \boldsymbol{\mu}_{n}(\mathbb{K}),
$$

the semidirect product of $G_{1}$ by $\boldsymbol{\mu}_{n}(\mathbb{K})$. Next,

$$
\mathrm{N} / \mathrm{N}^{\prime} \cong \mathbb{Z} / 2 \mathbb{Z} \text { if Condition }(\diamond) \text { holds, }
$$

and

$$
\mathrm{N}=\mathrm{N}^{\prime} \quad \text { otherwise. }
$$

Furthermore, $\mathrm{N}$ is self-normalizing in $G$.

(ii) Suppose that either char $(\mathbb{K})=0$ or char $(\mathbb{K})=p>2$ and $p$ does not divide $n$. Then the Lie algebra $\mathfrak{G}_{1}\left(\theta^{\varepsilon}\right)$ is self-normalizing in $\mathfrak{G}$.

In general, given any groups $G_{1} \leq G \leq \mathrm{GL}(n, \mathbb{K})$, one would like to know what is the normalizer $\mathrm{N}=\mathcal{N}_{G}\left(G_{1}\right)$, and then check whether $\mathrm{N}$ is selfnormalizing in $G$. For that we should be aware of the following two clear facts. First, the above answers about $\mathrm{N}$ strongly depend on that how $G_{1}$ is embedded in $G$. Second, we can have two isomorphic subgroups $G_{1}, G_{2}$ of $G$, but the corresponding normalizers $\mathrm{N}_{1}, \mathrm{~N}_{2}$ need not be isomorphic; cf. Lemma 4.1. In Section 4 we give some further remarks about the latter. In particular, there we consider some realizations of $\mathfrak{s p}(2 n, \mathbb{K})$ and $\mathfrak{s o}(2 n, \mathbb{K})$, within $\mathfrak{G}=$ $\mathfrak{s l}(2 n, \mathbb{K})$; thus we have symmetric pairs of Lie algebras $(\mathfrak{G}, \mathfrak{s p}(2 n, \mathbb{K}))$ and $(\mathfrak{G}, \mathfrak{s o}(2 n, \mathbb{K}))$. We also consider the corresponding pairs of groups. More precisely, we have five well known involutive automorphisms of $\mathfrak{G}$ : these are the above defined $\theta^{\varepsilon}$ and certain $\Theta^{\varepsilon}$ that will be recalled there, for $\varepsilon= \pm$, and the "minus transposing" $\vartheta: A \mapsto-A^{t}$. For $\theta$ being any of them, define the $\theta$-fixed point Lie algebra $\mathfrak{G}_{1}=\mathfrak{G}_{1}(\theta)=\mathfrak{G}^{\theta}$, and a subgroup $G_{1}=G_{1}(\theta)$ of $G=\mathrm{SL}(2 n, \mathbb{K})$, being the set of all $A \in G$ satisfying $A \theta(A)=-I$. Thus 
we have symmetric pairs $\left(\mathfrak{G}, \mathfrak{G}_{1}(\theta)\right)$ and the corresponding pairs of groups $\left(G, G_{1}(\theta)\right)$. Roughly speaking, we will see that under mild conditions on $\mathbb{K}$, the above theorem holds for any such $\theta$. Nevertheless, as it will be clear from what follows, the involutions $\theta^{\varepsilon}=\theta^{ \pm}$, and the corresponding $\mathfrak{G}_{1}\left(\theta^{\varepsilon}\right)$ and $G_{1}\left(\theta^{\varepsilon}\right)$, play the central role in our work. The reason is that these objects are particularly convenient for the computation of normalizers.

Concerning the structure of the normalizers $\mathrm{N}$, one more remark is in order. Note that when $\mathbb{K}$ is algebraically closed of characteristic zero, the "dictionary" algebraic group $\leftrightarrow$ its Lie algebra provides a solid ground to figure out what might be all this about. However even then we have to be careful. For an illustration of what can happen, let us present this instructive and definitive corollary which shows that for a very "tame" field, like $\mathbb{C}$ is, the question about the structure of $\mathrm{N}$ may not be trivial; see Lemmas 3.9 and 3.10 for its proof. Furthermore it clearly shows that the question "Whether Condition $(\diamond)$ holds?" is in general a delicate issue.

Corollary 0.2. Let $\mathbb{K}=\mathbb{C}$, and $G, G_{1}$ be as in the above theorem.

(i) (Orthogonal case). Suppose that $\varepsilon=1$ and $n$ is odd. Then we have the Condition $(\diamond)$, and thus $\mathrm{N} / G_{1} \cong \boldsymbol{\mu}_{2 n}(\mathbb{C})$. More precisely, for $G_{1}=S O(2 n, \mathbb{C})$, the normalizer $\mathrm{N}$ and the corresponding $\mathrm{N}^{\prime}$ we have

$$
\mathrm{N}^{\prime} \cong G_{1} \rtimes \mathbb{Z} / n \mathbb{Z} \quad \text { and } \quad \mathrm{N} / \mathrm{N}^{\prime} \cong \mathbb{Z} / 2 \mathbb{Z} .
$$

(ii) (Symplectic case). Suppose that $\varepsilon=-1$ and $n \equiv 2,3(\bmod 4)$. Then we do not have the Condition $(\diamond)$, and thus $\mathrm{N} / G_{1} \cong \boldsymbol{\mu}_{n}(\mathbb{C})$. More precisely, for $G_{1}=S p(2 n, \mathbb{C})$ and $\mathrm{N}$ we have

$$
\mathrm{N} \cong G_{1} \rtimes \mathbb{Z} / n \mathbb{Z} \text {. }
$$

Remark 0.3. (I) Notice that the only assumption we pose on $\mathbb{K}$ is $\operatorname{char}(\mathbb{K}) \neq 2$. So in particular we treat in our approach the class of finite groups of Lie type as well; and the corresponding Lie algebras defined over finite fields. As it is well known, a particularly pleasant fact in this "finite setting" is the possibility of counting/finite computations. So in particular one can often gain insight into a more complicated situation; e.g., concerning the geometry of orbits, when we have pairs $\left(G, G_{1}\right)$ and $\left(\mathfrak{g}, \mathfrak{g}_{1}\right)$ and want to understand a relationship between the (nilpotent) (co)adjoint orbits for $G$ and $G_{1}$, respectively. The latter remark puts a special emphasis on the case when the ground field $\mathbb{K}$ is finite; although all the results we have obtained hold for infinite $\mathbb{K}$ as well.

(II) There are a number of situations when we consider various (non)symmetric pairs of Lie algebras $\left(\mathfrak{g}, \mathfrak{g}_{1}\right)$ over a field of characteristic zero, where $\mathfrak{g}$ is semisimple and $\mathfrak{g}_{1}$ is reductive in it; or the corresponding pairs $\left(G, G_{1}\right)$ of groups. For example, in some branching problems, when we want to decompose the restriction $\rho_{\mid G_{1}}$ of an irreducible 
representation $\rho$ of $G$. Also, as we already noted, such pairs naturally arise while studying the geometry of orbits. For some important and/or recent results about various such pairs of Lie groups and Lie algebras see [HTW, Ko1, Ko2, BK, Kn, Ks, LS, V].

\section{Preliminaries}

1.1. Notation and conventions. In what follows, by $\mathbb{F}_{q}$ we denote the finite field with $q=p^{f}$ elements, where $p$ is a prime. Also, we assume that the reader is familiar with the basic facts about roots in finite fields; see, e.g., [Kob, Ch. II]. By $M_{n}(\mathbb{K})$ we denote the algebra of $n$-by- $n \mathbb{K}$-matrices. By $E_{i j}$, or $E_{i, j}$, we denote the matrix having 1 in the $(i, j)$-th place and 0 elsewhere. Define $\boldsymbol{s}=\boldsymbol{s}_{n}$ to be the $n$-by- $n$ matrix $\sum_{i=1}^{n} E_{i, n+1-i}$; i.e., $\boldsymbol{s}$ is a matrix with 1 on the skew diagonal and 0 elsewhere. For $\varepsilon \in\{ \pm 1\}$ we also define a $2 n$-by- $2 n$ matrix $S_{\varepsilon}=S_{+}$or $S_{-}$by

$$
S_{\varepsilon}=S_{n, \varepsilon}=\left(\begin{array}{cc}
0 & s \\
\varepsilon s & 0
\end{array}\right) .
$$

Suppose that $\mathfrak{g}$ is a $\mathbb{K}$-Lie algebra. By $B_{\mathfrak{g}}$ we denote its Killing form. For any $\theta \in$ Aut $\mathfrak{g}$, we define $\mathfrak{g}^{\theta}=\{X \in \mathfrak{g} \mid \theta(X)=X\}$, the fixed point algebra for $\theta$. Given a subalgebra $\mathfrak{s}$ of $\mathfrak{g}$, by $N_{\mathfrak{g}}(\mathfrak{s})$ and $C_{\mathfrak{g}}(\mathfrak{s})$ we denote the normalizer and centralizer, of $\mathfrak{s}$ in $\mathfrak{g}$, respectively. If $G$ is a group, and $S$ is its subgroup, by $\mathcal{N}_{G}(S)$ and $\mathcal{C}_{G}(S)$ we denote the normalizer and centralizer, of $S$ in $G$, respectively.

For an algebraic group $\boldsymbol{G}$ by $\mathcal{L}(\boldsymbol{G})$ we denote its Lie algebra. Suppose now that $\boldsymbol{G} \leq \mathrm{GL}(n, \overline{\mathbb{K}})$ is a (connected) algebraic $\mathbb{K}$-group, and let $\mathfrak{g}=\mathcal{L}(\boldsymbol{G}) \leq$ $\mathfrak{g l}(n, \overline{\mathbb{K}})$ be its Lie algebra. For such $\boldsymbol{G}$ and $\mathfrak{g}$ we have some interesting and well known actions/representations. First, we have the adjoint action Ad of $\boldsymbol{G}$ on itself. The corresponding orbits $\operatorname{Ad} \boldsymbol{G}(x), x \in \boldsymbol{G}$, are standardly called the conjugacy classes. Then we have the adjoint representation Ad of $\boldsymbol{G}$ on $\mathfrak{g}$, where the orbits are called the adjoint orbits. We also have the derived adjoint representation ad $: \mathfrak{g} \rightarrow \mathfrak{g l}(\mathfrak{g})$, which is a useful linear algebra-tool for studying the mentioned $\boldsymbol{G}$-orbits. Next, related to the representations Ad and ad on $\mathfrak{g}$, we have the coadjoint representations $\mathrm{Ad}^{*}$ and $\mathrm{ad}^{*}$ of $\boldsymbol{G}$ and $\mathfrak{g}$, respectively, on the dual $\mathfrak{g}^{*}$ of $\mathfrak{g}$. In particular, the $\mathrm{Ad}^{*}$-orbits are called the coadjoint orbits of $\boldsymbol{G}$.

Let $\boldsymbol{G}, \mathfrak{g}$ be as above, and $G=\boldsymbol{G}(\mathbb{K})$. Let $\mathfrak{g}(\mathbb{K})$ be the corresponding $\mathbb{K}$-structure of $\mathfrak{g}$. Then we have the adjoint representation of $G$ on $\mathfrak{g}(\mathbb{K})$. For two subalgebras $\mathfrak{s}_{1}, \mathfrak{s}_{2} \leq \mathfrak{g}(\mathbb{K})$ we say that they are $G$-conjugate, or just conjugate, if $\operatorname{Ad} g\left(\mathfrak{s}_{1}\right)=\mathfrak{s}_{2}$ for some $g \in G$. Analogously, we define the notion of $G$-conjugacy for subgroups of $G$.

1.2. Pairs of groups $\left(G, G_{1}\right)$. For our needs below let us introduce certain terminology. Let $\mathcal{M}$ be a $\mathbb{K}$-algebra. A $\mathbb{K}$-linear endomorphism $\delta$ 
of the additive group $\mathcal{M}$, which satisfies $\delta(x y)=\delta(y) \delta(x)$ for all $x, y \in \mathcal{M}$, will be called a $\mathbb{K}$-antiendomorphism of $\mathcal{M}$. A bijective $\mathbb{K}$-antiendomorphism is called a $\mathbb{K}$-antiautomorphism. Next suppose that $\mathcal{G}$ is an arbitrary group. As usual, by Aut $\mathcal{G}$ we denote the group of all automorphisms of $\mathcal{G}$. A map $\delta: \mathcal{G} \rightarrow \mathcal{G}$ will be called an antiendomorphism of $\mathcal{G}$ if $\delta\left(g_{1} g_{2}\right)=\delta\left(g_{2}\right) \delta\left(g_{1}\right)$ for all $g_{1}, g_{2} \in \mathcal{G}$. A bijective antiendomorphism is called an antiautomorphism of $\mathcal{G}$. By aAut $\mathcal{G}$ we denote the set of all antiautomorphisms of $\mathcal{G}$. Suppose now that $\mathcal{M}$ is a $\mathbb{K}$-algebra and $\mathcal{G}$ is a subgroup of the multiplicative monoid $\mathcal{M}^{\times}$. Given some $\mathbb{K}$-antiendomorphism $\delta$ of $\mathcal{M}$ such that $\delta(\mathcal{G}) \subseteq \mathcal{G}$ we say that $\mathcal{G}$ is $\delta$-stable.

Now we are going to explain what kind of groups $G$ and their subgroups $G_{1}$ we would like to study in general. But first we need a basic preliminary observation concerning certain endomorphisms on matrices.

We will consider certain $\mathbb{K}$-antiendomorphisms $A \mapsto A^{\sharp}$ of $M_{n}(\mathbb{K})$ which moreover map the identity matrix to itself. That is, our maps will satisfy the following two conditions:

$(\sharp 1) I^{\sharp}=I$;

$(\sharp 2)(A B)^{\sharp}=B^{\sharp} A^{\sharp}$, for all $A, B \in M_{n}(\mathbb{K})$.

We will also consider some $\mathbb{K}$-linear endomorphisms $\theta$ of the additive group $M_{n}(\mathbb{K})$ satisfying the following two conditions:

(ө1) $\theta(A B)=-\theta(B) \theta(A)$, for all $A, B \in M_{n}(\mathbb{K})$;

$(\boldsymbol{\theta 2})$ There exists a regular $A_{0}$ such that $\theta\left(A_{0}\right)$ is regular as well.

Somewhat roughly stated, the following lemma explains that the two ways for choosing a $\mathbb{K}$-endomorphism of $M_{n}(\mathbb{K})$ are in fact equivalent. Let us emphasize here that both possibilities will be useful for us, depending on concrete situations we have. An easy proof is omitted.

Lemma 1.1. Suppose we have a $\mathbb{K}$-endomorphism $A \mapsto A^{\sharp}$ of $M_{n}(\mathbb{K})$

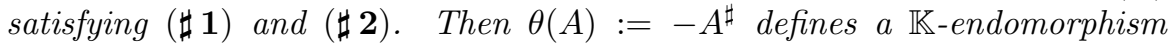
satisfying $(\boldsymbol{\theta 1})$ and $(\boldsymbol{\theta} \mathbf{2})$.

Conversely, suppose we have a $\mathbb{K}$-endomorphism $\theta$ of $M_{n}(\mathbb{K})$ satisfying $(\boldsymbol{\theta 1})$ and $(\boldsymbol{\theta 2})$. Then $A \mapsto A^{\sharp}:=-\theta(A)$ defines a $\mathbb{K}$-endomorphism satisfying $(\sharp 1)$ and $(\sharp 2)$.

REMARK 1.2. It is a natural question of how many $\mathbb{K}$-endomorphisms $A \mapsto A^{\sharp}$ of a concrete group $G$ we will have. Roughly speaking, the answer is that often we can expect to have a number of such maps. Let us give an argument for that statement. For that purpose, let $\mathcal{M}$ and a group $\mathcal{G} \subseteq \mathcal{M}^{\times}$ be as in the first paragraph of the present subsection. Note that then for an arbitrary $\delta \in$ aAut $\mathcal{G}$ we have both $\delta^{-1} \in$ aAut $\mathcal{G}$ and $\delta(e)=e$; where $e=e_{\mathcal{G}}$ is the identity of $\mathcal{G}$.

Fix now some $\delta \in$ aAut $\mathcal{G}$, and then define a map $\Upsilon:$ Aut $G \rightarrow$ aAut $G$, given by $\Upsilon(\alpha)=\alpha \circ \delta$, for $\alpha \in$ Aut $\mathcal{G}$. 
Claim. The map $\Upsilon$ is a well defined bijection.

Proof. First note that for $\alpha \in \operatorname{Aut} \mathcal{G}$ and $g_{1}, g_{2} \in \mathcal{G}$ we have

$$
\Upsilon(\alpha)\left(g_{1} g_{2}\right)=\alpha\left(\delta\left(g_{2}\right) \delta\left(g_{1}\right)\right)=\Upsilon(\alpha)\left(g_{2}\right) \Upsilon(\alpha)\left(g_{1}\right) ;
$$

i.e., $\Upsilon(\alpha) \in$ aAut $\mathcal{G}$. So $\Upsilon$ is well defined.

Next, using that $\delta$ is bijective, it is clear that $\Upsilon$ is injective. It remained to show that $\Upsilon$ is moreover surjective. For that purpose let $\widetilde{\alpha} \in$ aAut $\mathcal{G}$ be arbitrary. Then define a map $\alpha: \mathcal{G} \rightarrow \mathcal{G}$ by $\alpha=\widetilde{\alpha} \circ \delta^{-1}$. Using the above observation that $\delta^{-1} \in$ aAut $\mathcal{G}$, it immediately follows that $\alpha \in$ Aut $\mathcal{G}$. Next we note that $\Upsilon(\alpha)=\widetilde{\alpha}$. Therefore $\Upsilon$ is indeed surjective, as we had to see.

Let now $G \leq \mathrm{GL}(n, \mathbb{K})$ be any group. Suppose we have a $\mathbb{K}$ endomorphism $A \mapsto A^{\sharp}$ of $M_{n}(\mathbb{K})$ satisfying ( $\left.\sharp \mathbf{1}\right)$ and ( $\left.\sharp 2\right)$. Next suppose that $G$ is $\sharp$-stable. We also assume that the following condition on the center of $G$ holds:

$$
\mathcal{Z}(G) \subseteq \mathbb{K}^{\times} I
$$

Notice that this condition is a reasonable one, and it holds for a number of groups $G$. Also, concerning it, observe that for any subgroup $S \leq G$ we have $\mathcal{Z}(G) \leq \mathcal{C}_{G}(S)$.

In Section 3 below and [Š5] we will emphasize the role of certain $\mathbb{K}$ endomorphisms of $M_{n}(\mathbb{K})$ while studying various pairs of groups; these will be written as $A \mapsto A^{\sharp}$. Here we would first like to present a slightly different, and more general, point of view. Roughly speaking, a part on the "group side" of our setting is to consider certain pairs of groups $\left(G, G_{1}\right)$, where $G_{1} \leq G \leq$ $\mathrm{GL}(n, \mathbb{K})$. To be more precise, suppose we have a subgroup $G \leq \mathrm{GL}(n, \mathbb{K})$ and a map $\theta: G \rightarrow \operatorname{GL}(n, \mathbb{K})$ satisfying

$$
\theta\left(g_{1} g_{2}\right)=-\theta\left(g_{2}\right) \theta\left(g_{1}\right), \quad g_{1}, g_{2} \in G \text {. }
$$

Note that then in particular $\theta(I)=-I$ and $\theta\left(g^{-1}\right)=\theta(g)^{-1}$, for all $g$; cf. Lemma 1.1. Next define

$$
G_{1}=\{g \in G \mid \theta(g) g=-I\}
$$

note also here that $\theta(g) g=-I$ if and only if $g \theta(g)=-I$. It is clear that $G_{1}$ is a subgroup of $G$; cf. Lemma 4.2 below, and see [Wa, Sect. 2] or [Wl, Sect. $2]$ for the meaning of the so-called norm group.

Suppose now that $\boldsymbol{G} \leq \mathrm{GL}(n, \overline{\mathbb{K}})$ is a connected affine group. Let $\mathfrak{G}=$ $\mathcal{L}(\boldsymbol{G})$ and $G=\boldsymbol{G}(\mathbb{K})$. Let also $\mathfrak{g}=\mathfrak{G}(\mathbb{K})$, a "corresponding" $\mathbb{K}$-structure of $\mathfrak{G}$. Let now $\theta$ be a $\mathbb{K}$-endomorphism of $M_{n}(\mathbb{K})$ satisfying $(\boldsymbol{\theta 1})$ and $(\boldsymbol{\theta 2})$; recall that then $\theta(A)$ is regular for every regular $A$. Suppose also that $\theta(\mathfrak{g})=\mathfrak{g}$. 
Hence it follows that $\theta=\theta_{\mid \mathfrak{g}} \in$ Aut $\mathfrak{g}$; cf. Lemma 1.4 below. A setting in which we are interested is the following one:

$$
\begin{aligned}
& \text { The pair of groups }\left(G, G_{1}\right) \text {, and } \\
& \text { the corresponding pair of Lie algebras }\left(\mathfrak{g}, \mathfrak{g}_{1}\right) \text {; }
\end{aligned}
$$

where $G_{1}$ is defined as in (1.2), and $\mathfrak{g}_{1}=\mathfrak{g}^{\theta}$.

Now when we have these $G_{1}$ and $\mathfrak{g}_{1}$, it is natural to ask how they are related. Although this is not obvious, and we do not know an argument in general, the answer is in many cases just as one would like to have it. Namely, as for $G$ and $\mathfrak{g}$, we will have $G_{1}=G_{1}(\mathbb{K})$ and $\mathfrak{g}_{1}=\mathfrak{G}_{1}(\mathbb{K})$, for certain affine group $\boldsymbol{G}_{1} \leq \boldsymbol{G}$ and its Lie algebra $\mathfrak{G}_{1}=\mathcal{L}\left(\boldsymbol{G}_{1}\right)$. Here we will confine ourselves only to the case $\mathbb{K}=\mathbb{C}$, and $\boldsymbol{G} \leq \mathrm{GL}(n, \mathbb{C})$ a connected reductive group. Define, as before, $\mathfrak{G}=\mathcal{L}(\boldsymbol{G})$, and let $\theta$ be a $\mathbb{C}$-endomorphism of $M_{n}(\mathbb{C})$ satisfying $(\boldsymbol{\theta 1})$ and $(\boldsymbol{\theta 2})$. Let $\boldsymbol{G}_{1}$ be a subgroup of $\boldsymbol{G}$, defined as in (1.2), and consider a Lie algebra $\mathfrak{G}_{1}=\mathfrak{G}^{\theta}$. Then we have the following result.

Proposition 1.3. The subgroup $\boldsymbol{G}_{1}$ is an algebraic group, and $\mathfrak{G}_{1}$ is its Lie algebra.

Proof. Let $\boldsymbol{G}$ and $\mathfrak{G}$ be as above. Take a maximal torus $\boldsymbol{T} \leq \boldsymbol{G}$ such that every $h \in \boldsymbol{T}$ is diagonal. For every such $h$ we can find a sufficiently big $m \in \mathbb{N}$, depending on $h$, and $g \in \boldsymbol{G}$ sufficiently close to $I$ so that $g^{m}=h$ and $g=\exp _{\boldsymbol{G}} X$, for some $X \in \mathfrak{G}$. Thus also $\exp _{\boldsymbol{G}} m X=h$; i.e., $h \in \exp _{\boldsymbol{G}} \mathfrak{G}$. The set $\boldsymbol{G}_{s}$, of all semisimple elements of $\boldsymbol{G}$, is equal to $\bigcup_{g \in \boldsymbol{G}} g \boldsymbol{T} g^{-1}$, and therefore is dense in $\boldsymbol{G}$; see [Bo, Thm. 11.10]. Thus we have the following claim.

Claim. The set $\exp _{\boldsymbol{G}} \mathfrak{G}$ is dense in $\boldsymbol{G}$.

Assume now that we know the values of $\theta$ on $\mathfrak{G}$. Then for $g=\exp _{G} X$, $X \in \mathfrak{G}$, we immediately deduce that

$$
\theta(g)=-\exp _{\boldsymbol{G}}-\theta(X) .
$$

Thus, taking into account the above Claim, we know how to compute $\theta(g)$, for every $g \in \boldsymbol{G}$.

To prove the proposition we only have to show that $\mathcal{L}\left(\boldsymbol{G}_{1}\right)=\mathfrak{G}_{1}$. For the inclusion from left to right, take any $X \in \mathcal{L}\left(\boldsymbol{G}_{1}\right)$ and $t \in \mathbb{C}$, and define $g_{t}=\exp _{\boldsymbol{G}}(t X)$. Using (1.3) and the fact that $g_{t} \in \boldsymbol{G}_{1}$, it follows at once that $\exp _{G} t X=\exp _{G} t \theta(X)$. The last equality is further equivalent to

$$
\left(\exp _{G} t X-I\right) / t=\left(\exp _{G} t \theta(X)-I\right) / t
$$

Take a limit when $t \rightarrow 0$. We obtain that $X=\theta(X)$; i.e., $X \in \mathfrak{G}_{1}$. The opposite inclusion is along the same lines.

Let us proceed with more details, and in particular explain a general procedure for obtaining the maps $\theta$ as above. Suppose $\varphi$ is a $\mathbb{K}$-antiendomorphism of $M_{n}(\mathbb{K})$ satisfying $\varphi^{k}=1$, for some $k \geq 2$. Note that then $\varphi$ is 
moreover a $\mathbb{K}$-antiautomorphism. Also, $\varphi(I)=I$ and $\varphi\left(A^{-1}\right)=\varphi(A)^{-1}$, for every regular $A$. Next, let $G$ and $\mathfrak{g}$ be as in the paragraph containing $(\star)$, and assume that $\varphi(\mathfrak{g})=\mathfrak{g}$. Pick any $\nu \in G$ such that it satisfies the following:

(०) $\varphi(\nu) \nu^{-1} \in \mathcal{C}_{G}(\mathfrak{g})$.

Then define $A^{\sharp}=\nu \varphi(A) \nu^{-1}$, for all $A$, and $\theta(A)=-A^{\sharp}$; i.e.,

$$
\theta=-\operatorname{Int} \nu \circ \varphi
$$

Thus we in particular have

$$
\begin{aligned}
\theta^{2}(A) & =-\nu \varphi(\theta(A)) \nu^{-1} \\
& =-\nu\left(\varphi\left(\nu^{-1}\right) \varphi^{2}(A) \varphi(\nu) \varphi(-I)\right) \nu^{-1} \\
& =\varphi^{2}(A) ;
\end{aligned}
$$

here we use that $\varphi^{2}(A) \in \mathfrak{g}, \varphi(-I)=-I$ and the condition (o). Hence also $\theta^{3}(A)=-\nu \varphi^{3}(A) \nu^{-1}$; and, by induction,

$$
\theta^{k}(A)= \begin{cases}A & \text { if } k \text { even, } \\ -\nu A \nu^{-1} & \text { if } k \text { odd } .\end{cases}
$$

The following basic observation is now more or less an easy consequence of the above.

Lemma 1.4. The map $\theta$ satisfies both $(\boldsymbol{\theta 1})$ and $(\boldsymbol{\theta 2})$. Moreover, $\theta=\theta_{\mid \mathfrak{g}}$ is an automorphism of $\mathfrak{g}$ satisfying $\theta^{k}=1_{\mathfrak{g}}$, provided that $k \geq 2$ is even. In particular, for $k=2, \theta$ is an involutive automorphism of $\mathfrak{g}$; i.e., $(\mathfrak{g}, \theta)$ is a symmetric Lie algebra.

Remark 1.5. Suppose that $\varphi^{k}=1$ and $k$ is odd. Suppose also that moreover $\nu \in \mathcal{C}_{G}(\mathfrak{g})$; cf. Remark 1.8 below. Note that then $\theta=\theta_{\mid \mathfrak{g}}=-\varphi$ is again an automorphism of $\mathfrak{g}$, but at the same time now we have $\theta^{k}=-1_{\mathfrak{g}}$.

For more details about the example which follows see Section 3 below, and [ک̌S5, Sect. 2].

ExAmPLE 1.6. For $m=2 n>2$ define block-matrices $J_{ \pm}^{\text {even }}=\left(\begin{array}{cc}0 & I \\ \pm & 0\end{array}\right) \in$ $M_{m}(\mathbb{K})$, and for $m=2 n+1$ define a block-matrix $J^{\text {odd }}=\left(\begin{array}{cc}1 & 0 \\ 0 & J_{+}^{\text {even }}\end{array}\right) \in M_{m}(\mathbb{K})$. Now for any $A \in M_{m}(\mathbb{K})$ let $\varphi(A)=A^{t}$, the transpose of $A$. Also define $A^{\sharp}=J^{t} \varphi(A) J=J^{t} A^{t} J$ and $\theta(A)=-A^{\sharp}$, where $J$ is either $J_{ \pm}^{\text {even }}$ or $J^{\text {odd }}$. Note here that for $\nu=J=J_{ \pm}^{\text {even }}$ we have $\varphi(\nu) \nu^{-1}=\nu^{t} \nu^{-1}= \pm I$, while for $\nu=J=J^{\text {odd }}, \nu^{t} \nu^{-1}=I$. Note also that if we put $G=\operatorname{SL}(m, \mathbb{K})$ and $\mathfrak{g}=\mathfrak{s l}(m, \mathbb{K})$, then $\mathcal{C}_{G}(\mathfrak{g})=\boldsymbol{\mu}_{m}(\mathbb{K}) I$. Thus in particular the condition $\varphi(\nu) \nu^{-1} \in \mathcal{C}_{G}(\mathfrak{g})$ holds. Explicitly, if $m=2 n$, then

$$
\left(\begin{array}{cc}
X & Y \\
Z & T
\end{array}\right)=A \longmapsto \theta(A)=\left(\begin{array}{cc}
-T^{t} & \mp Y^{t} \\
\mp Z^{t} & -X^{t}
\end{array}\right)
$$


here $X, Y, Z, T \in M_{n}(\mathbb{K})$. Also, if $m=2 n+1$, then

$$
\left(\begin{array}{ccc}
a & \boldsymbol{r}_{\mathbf{1}} & \boldsymbol{r}_{\mathbf{2}} \\
\boldsymbol{c}_{\mathbf{1}} & X & Y \\
\boldsymbol{c}_{\mathbf{2}} & Z & T
\end{array}\right)=A \longmapsto \theta(A)=\left(\begin{array}{ccc}
-a & -\boldsymbol{c}_{\mathbf{2}}{ }^{t} & -\boldsymbol{c}_{\mathbf{1}}{ }^{t} \\
-\boldsymbol{r}_{\mathbf{2}}{ }^{t} & -T^{t} & -Y^{t} \\
-\boldsymbol{r}_{\mathbf{1}}{ }^{t} & -Z^{t} & -X^{t}
\end{array}\right)
$$

here $a \in \mathbb{K}, \boldsymbol{r}_{\mathbf{1 , 2}} \in M_{1 n}(\mathbb{K}), \boldsymbol{c}_{\mathbf{1 , 2}} \in M_{n 1}(\mathbb{K})$ and $X, Y, Z, T \in M_{n}(\mathbb{K})$.

We conclude this subsection with one more example which is at the same time very instructive. In particular, it indicates that we will have an analogous structural result about the normalizer $\mathcal{N}_{G}\left(G_{1}\right)$, for various other pairs of groups $\left(G, G_{1}\right)$.

EXAmPLE 1.7. Consider a $\mathbb{K}$-endomorphism of $M_{3}(\mathbb{K})$ given by

$$
\theta\left(\begin{array}{lll}
d_{1} & x_{1} & x_{3} \\
y_{1} & d_{2} & x_{2} \\
y_{3} & y_{2} & d_{3}
\end{array}\right)=\left(\begin{array}{ccc}
-d_{3} & x_{2} & -x_{3} \\
y_{2} & -d_{2} & x_{1} \\
-y_{3} & y_{1} & -d_{1}
\end{array}\right)
$$

cf. [ک̌S4, Rmk. 3.4]. An easy checking shows that $\theta$ satisfies both $(\boldsymbol{\theta 1})$ and $(\boldsymbol{\theta 2})$, and $\theta^{2}=1$. Thus, in particular, $\theta \in \operatorname{Aut} \mathfrak{g l}(3, \mathbb{K})$. If we put $G=\operatorname{SL}(3, \mathbb{K})$ and take $G_{1}$ to be as in (1.2), then one can show that $G_{1}=\operatorname{PSL}(2, \mathbb{K})$.

Let $\nu=\operatorname{diag}(-1,1,-1) \in G$. Let $\varphi(A)=A^{\tau}$, the skew transpose of $A$; see Lemma 4.2. Now we have $\theta=-\operatorname{Int} \nu \circ \varphi$. In other words, the above defined $\theta$ is also obtained via our general construction.

For the obtained pair of groups $\left(G, G_{1}\right)$, one can prove the following claim. Although elementary, it is not quite easy; the details will appear elsewhere.

Claim. For the normalizer $\mathrm{N}=\mathcal{N}_{G}\left(G_{1}\right)$ we have

$$
\mathrm{N} \cong G_{1} \rtimes \mu_{3}(\mathbb{K})
$$

and this is a self-normalizing subgroup of $G$.

Remark 1.8. Note that the condition $\nu \in \mathcal{C}_{G}(\mathfrak{g})$ implies $\varphi(\nu) \nu^{-1} \in$ $\mathcal{C}_{G}(\mathfrak{g})$. But, as we saw in the previous example, the converse in general does not hold. Thus, loosely speaking, the mentioned condition provides more maps $\theta$ than the former one.

REMARK 1.9. In [క̌́6] we will explain how to obtain some non-reductive pairs of groups on which our structural results can be applied. More precisely, we will treat some pairs that might be called parabolic pairs. For that we consider $G=\mathrm{SL}(2 n, \mathbb{K})$ or $\mathrm{GL}(2 n, \mathbb{K})$, and certain conveniently chosen antiautomorphisms $A \mapsto A^{\sharp}$ of $G$. Thus we obtain the corresponding subgroup $G_{1}$ of $G$. Let then $Q$ be any $\sharp$-stable standard parabolic subgroup of $G$, and $Q_{1}=Q \cap G_{1}$, a parabolic subgroup of $G_{1}$. For such pairs $\left(Q, Q_{1}\right)$ we have analogous result about the structure of the normalizer $\mathcal{N}_{Q}\left(Q_{1}\right)$. 


\section{Pairs of Lie Algebras}

Let $\mathfrak{g}$ be a $\mathbb{K}$-Lie algebra. Consider a pair $\left(\mathfrak{g}, \mathfrak{g}_{1}\right)$, where $\mathfrak{g}_{1}$ is a proper subalgebra of $\mathfrak{g}$. Having in mind the classical situation in the zero characteristic, we will have the following terminology. The pair $\left(\mathfrak{g}, \mathfrak{g}_{1}\right)$ is symmetric if $\mathfrak{g}_{1}=\mathfrak{g}^{\theta}$, for some involutive automorphism $\theta$ of $\mathfrak{g}$, and nonsymmetric if $\mathfrak{g}_{1} \neq \mathfrak{g}^{\theta}$ for any such $\theta$; we also say that $\mathfrak{g}_{1}$ is a (non)symmetric subalgebra of $\mathfrak{g}$. Analogously as for $\operatorname{char}(\mathbb{K})=0$, we have the following about the symmetric pairs; the proof is straightforward and will be omitted.

Proposition 2.1. Let a pair $\left(\mathfrak{g}, \mathfrak{g}_{1}\right)$ be symmetric, via $\theta$, and define $\mathfrak{p}=$ $\{x \in \mathfrak{g} \mid \theta(x)=-x\}$.

(i) We have $\left[\mathfrak{g}_{1}, \mathfrak{p}\right] \subseteq \mathfrak{p},[\mathfrak{p}, \mathfrak{p}] \subseteq \mathfrak{g}_{1}$ and a $B_{\mathfrak{g}}$-orthogonal direct sum $\mathfrak{g}=$ $\mathfrak{g}_{1} \oplus \mathfrak{p}$.

(ii) If $B_{\mathfrak{g}}$ is nondegenerate, then both restrictions of $B_{\mathfrak{g}}$, to $\mathfrak{g}_{1}$ and to $\mathfrak{p}$, are nondegenerate too.

Consider now a (nonsymmetric) pair $\left(\mathfrak{g}, \mathfrak{g}_{1}\right)$. Let $\boldsymbol{\beta}$ denotes the restriction of $B_{\mathfrak{g}}$ to $\mathfrak{g}_{1}$. In what follows we always assume that such pairs of Lie algebras satisfy the following:

(C) $\boldsymbol{\beta}$ is a nondegenerate form.

To avoid certain trivialities we also assume:

(P) $\mathfrak{g}_{1}$ is not an ideal of $\mathfrak{g}$.

For $\left(\mathfrak{g}, \mathfrak{g}_{1}\right)$ as above, and their duals $\mathfrak{g}^{*}$ and $\mathfrak{g}_{1}^{*}$, let $r: \mathfrak{g}^{*} \rightarrow \mathfrak{g}_{1}^{*}$ be the restriction map. By $\kappa: \mathfrak{g} \rightarrow \mathfrak{g}^{*}$ we denote the Killing homomorphism. Define also an isomorphism $\kappa_{1}: \mathfrak{g}_{1} \rightarrow \mathfrak{g}_{1}^{*}, \kappa_{1}\left(x_{1}\right)\left(y_{1}\right)=\boldsymbol{\beta}\left(x_{1}, y_{1}\right)$, for $x_{1}, y_{1} \in \mathfrak{g}_{1}$. Then define a map $\pi: \mathfrak{g} \rightarrow \mathfrak{g}_{1}$ such that $\kappa_{1} \circ \pi=r \circ \kappa$. Note that, by definition of $\pi$, for $x \in \mathfrak{g}$ and $x_{1} \in \mathfrak{g}_{1}$ we have

$$
B_{\mathfrak{g}}\left(x, x_{1}\right)=\boldsymbol{\beta}\left(\pi(x), x_{1}\right) .
$$

As we will see below, this $\pi$ is a $\mathfrak{g}_{1}$-module homomorphism; we call it the homomorphism associated to $\left(\mathfrak{g}, \mathfrak{g}_{1}\right)$. Next define a subspace $\mathfrak{p} \leq \mathfrak{g}$ as $\mathfrak{p}=$ $\operatorname{ker} \pi$. The following is a straightforward generalization of [ک̌S3, Prop. 3.2] to the present situation. For completeness (and because the proof given in [ $\breve{S} 3]$ is somewhat sketchy) we provide more details.

Proposition 2.2. Let $\left(\mathfrak{g}, \mathfrak{g}_{1}\right)$ be a pair of Lie algebras as above. Then:

(i) $\pi_{\mid \mathfrak{g}_{1}}=1_{\mathfrak{g}_{1}}$.

(ii) We have a Killing-orthogonal direct sum decomposition

$$
\mathfrak{g}=\mathfrak{g}_{1} \oplus \mathfrak{p} .
$$

(iii) $\left[\mathfrak{g}_{1}, \mathfrak{p}\right] \subseteq \mathfrak{p}$.

(iv) $\pi$ is a $\mathfrak{g}_{1}$-module homomorphism. 
(v) If $B_{\mathfrak{g}}$ is nondegenerate, then the restriction of $B_{\mathfrak{g}}$ to $\mathfrak{p}$ is nondegenerate as well.

Proof. (i) For $x_{1} \in \mathfrak{g}_{1}$ define $w=\pi\left(x_{1}\right)-x_{1}$. Using (2.1), we have $B_{\mathfrak{g}}\left(w, \mathfrak{g}_{1}\right)=\boldsymbol{\beta}\left(w, \mathfrak{g}_{1}\right)=0$. By (C), $w=0$. (ii) Write any $x \in \mathfrak{g}$ as $x=$ $\pi(x)+x-\pi(x)$, and note that by (i) and the definition of $\pi$ we have $\pi(x) \in \mathfrak{g}_{1}$ and $x-\pi(x) \in \mathfrak{p}$; thus $\mathfrak{g}=\mathfrak{g}_{1}+\mathfrak{p}$. For $w \in \mathfrak{g}_{1} \cap \mathfrak{p}$, by (2.1) and (C), again, we have $\boldsymbol{\beta}\left(w, \mathfrak{g}_{1}\right)=0$ and so $w=0$. Also, $B_{\mathfrak{g}}\left(\mathfrak{p}, \mathfrak{g}_{1}\right)=\boldsymbol{\beta}\left(\pi(\mathfrak{p}), \mathfrak{g}_{1}\right)=0$. Thus $\mathfrak{g}=\mathfrak{g}_{1} \oplus \mathfrak{p}$, a Killing-orthogonal direct sum. (iii) Take any $x_{1} \in \mathfrak{g}_{1}$ and $p \in \mathfrak{p}$, and write, by (ii), $\left[x_{1}, p\right]=y_{1}+q$, where $y_{1} \in \mathfrak{g}_{1}$ and $q \in \mathfrak{p}$. If we would have $y_{1} \neq 0$, then it would follow that $\boldsymbol{\beta}\left(u_{1}, y_{1}\right) \neq 0$ for some $u_{1} \in \mathfrak{g}_{1}$. But also, by the invariance of $B_{\mathfrak{g}}$,

$$
\boldsymbol{\beta}\left(u_{1}, y_{1}\right)=B_{\mathfrak{g}}\left(u_{1},\left[x_{1}, p\right]-q\right)=B_{\mathfrak{g}}\left(\left[u_{1}, x_{1}\right], p\right)=0 ;
$$

a contradiction. (iv) We have to see that $\pi\left(\left[y_{1}, x\right]\right)=\left[y_{1}, \pi(x)\right]$, for all $y_{1} \in \mathfrak{g}_{1}$ and $x \in \mathfrak{g}$. For that, write $x=x_{1}+p$, with $x_{1} \in \mathfrak{g}_{1}$ and $p \in \mathfrak{p}$. Then, clearly, $\left[y_{1}, \pi(x)\right]=\left[y_{1}, x_{1}\right]$. Also, by (i) and (iii), $\pi\left(\left[y_{1}, x\right]\right)=\pi\left(\left[y_{1}, x_{1}\right]\right)+\pi\left(\left[y_{1}, p\right]\right)=$ $\left[y_{1}, x_{1}\right]$ as well. (v) This is obvious.

The following simple result gives a characterization of self-normalizedness of $\mathfrak{g}_{1}$ in $\mathfrak{g}$, and a characterization of symmetric pairs. It is a direct generalization of Lemmas 3.3 and 3.4 in [Š3]; and its proof is essentially the same as there.

LEMMA 2.3. Let $\left(\mathfrak{g}, \mathfrak{g}_{1}\right)$ be a pair of Lie algebras as above.

(i) This pair is symmetric if and only if $[\mathfrak{p}, \mathfrak{p}] \subseteq \mathfrak{g}_{1}$.

(ii) If $B_{\mathfrak{g}}$ is nondegenerate, then the following are equivalent: (a) $\mathfrak{p}^{\mathfrak{g}_{1}}=0$; (b) $N_{\mathfrak{g}}\left(\mathfrak{g}_{1}\right)=\mathfrak{g}_{1} ;$ (c) $\left[\mathfrak{g}_{1}, \mathfrak{p}\right]=\mathfrak{p}$.

Let $\left(\mathfrak{g}, \mathfrak{g}_{1}\right)$ be a pair, and $\mathfrak{g}=\mathfrak{g}_{1} \oplus \mathfrak{p}$ the corresponding decomposition. The next basic results, the lemma and proposition below, are concerned with the problem of how the coadjoint orbits on $\mathfrak{g}^{*}$ and $\mathfrak{g}_{1}^{*}$ are related. These are Lemma 1.6 and Proposition 1.8 of [Š 4$]$ in the present, more general, setting. For the convenience of the reader we recall the statements; after obvious minor changes, the arguments given in [ک̌S4] also remain valid here.

Lemma 2.4. (i) The subspaces $\pi([\mathfrak{p}, \mathfrak{p}])$ and $\mathfrak{p}+[\mathfrak{p}, \mathfrak{p}]$ are ideals of $\mathfrak{g}_{1}$ and $\mathfrak{g}$, respectively.

(ii) If $\mathfrak{g}$ or $\mathfrak{g}_{1}$ is simple, then there is no $0 \neq \gamma \in \mathfrak{g}^{*}$ satisfying both $\gamma_{\mid \mathfrak{p}}=0$ and $\mathfrak{g} \cdot \gamma=\mathfrak{g}_{1} \cdot \gamma$.

Proposition 2.5. Define the trivial extension $\varepsilon: \mathfrak{g}_{1}^{*} \rightarrow \mathfrak{g}^{*}$ by $\varepsilon(\mu)_{\mid \mathfrak{p}}=0$. Then, for an arbitrary $\mu \in \mathfrak{g}_{1}^{*}$, we have

$$
\mathfrak{g} \cdot \varepsilon(\mu) \cap \mathfrak{g}_{1}^{*}=\mathfrak{g}_{1} \cdot \mu .
$$


To the end of this section we discuss the condition $(\mathbf{C})$; as we will see below, outside of the case $\operatorname{char}(\mathbb{K})=0$ this is a delicate issue. For what follows first suppose that $\mathfrak{G} \leq \mathfrak{s l}(n, \mathbb{C})$ is a simple complex Lie algebra. Then there exists a certain nonzero $c=c(\mathfrak{G}) \in \mathbb{Z}$ such that $B_{\mathfrak{G}}(X, Y)=c \operatorname{Tr}(X Y)$, for all $X, Y \in \mathfrak{G}$. Choose now a Chevalley basis $\mathcal{C}$ of $\mathfrak{G}$, and define a $\mathbb{Z}$-Lie algebra $\mathfrak{G}_{\mathbb{Z}}=\operatorname{span}_{\mathbb{Z}} \mathcal{C}$; see $[\mathrm{Ch}]$. Let $\mathbb{K}$ be a field of odd characteristic $p$, and define a $\mathbb{K}$-Lie algebra $\mathfrak{G}_{\mathbb{K}}=\mathfrak{G}_{\mathbb{Z}} \otimes_{\mathbb{Z}} \mathbb{K}$, a Chevalley algebra corresponding to $\mathfrak{G}$ and $\mathbb{K}$. Clearly, we have $B_{\mathfrak{G}_{\mathbb{K}}}(X, Y)=c \operatorname{Tr}(X Y)$, for all $X, Y \in \mathfrak{G}_{\mathbb{K}}$; here of course we consider $c$ to be an element of $\mathbb{F}_{p}$ (recall that the later equality is quite easy to show when $\mathbb{K}$ is algebraically closed of characteristic zero; see Exercise 18 for Ch. I, $\S 6$ in [B1]).

As a consequence of the above we in particular note the following. For an arbitrary field $\mathbb{K}$ and $\mathfrak{g}=\mathfrak{s l}(n, \mathbb{K})$, we have $B_{\mathfrak{g}}(x, y)=2 n \operatorname{Tr}(x y)$, for all $x, y \in \mathfrak{g}$. Also, if we take an ordered basis $\Lambda$ of $\mathfrak{g}$, where

$$
\Lambda=\left(E_{12}, E_{21}, \ldots, E_{i j}, E_{j i}, \ldots ; H_{1}, \ldots, H_{n-1}\right),
$$

and $H_{i}=E_{i i}-E_{i+1, i+1}$, then the matrix $B_{\mathfrak{g}}(\Lambda)$, corresponding to $B_{\mathfrak{g}}$ with respect to $\Lambda$, has a block-diagonal form $\operatorname{diag}(\mathcal{E}, \ldots, \mathcal{E} ; \mathcal{H})$. Here we have $n(n-$ $1) / 2$ blocks $\mathcal{E}=\left(\begin{array}{cc}0 & 2 n \\ 2 n & 0\end{array}\right)$ and one block $\mathcal{H}$ of size $n-1$ having $4 n$ on the main diagonal, $-2 n$ on the first diagonals next to the main one, and 0 elsewhere. Since $\operatorname{det} \mathcal{H}=n(2 n)^{n-1}$,

$$
\operatorname{det} B_{\mathfrak{g}}(\Lambda)=(-1)^{n(n-1) / 2} 2^{n^{2}-1} n^{n(n+1) / 2} .
$$

For our purposes we state explicitly this straightforward consequence of the above expression.

OBSERVATION. Let $n \in \mathbb{N}$, and $\mathbb{K}$ be any field such that either char $(\mathbb{K})=$ 0 , or $\operatorname{char}(\mathbb{K})=p>2$ and $p$ does not divide $n$. Then, for $\mathfrak{g}=\mathfrak{s l}(n, \mathbb{K})$, the Killing form $B_{\mathfrak{g}}$ is nondegenerate.

REMARK 2.6. The above observation is in fact more or less a special case of a more general result. Namely, suppose for the moment that $\mathbb{K}=\overline{\mathbb{K}}$, and $G$ is a simple algebraic group. Let $\mathfrak{G}$ be its Lie algebra. A well known result ([SS, Ch. I, §4]; see also [Ca, Sect. 1.13]) states that the Killing form $B_{\mathfrak{G}}$ is nondegenerate provided that $\operatorname{char}(\mathbb{K})=0$ or $\operatorname{char}(\mathbb{K})=p>h$, where $h$ is the Coxeter number of $G$; recall that the Coxeter number of $\mathfrak{s l}(n)$ is $n$.

To illustrate clearly the difference between the cases $\operatorname{char}(\mathbb{K})=0$ and $>0$, while discussing the condition $(\mathbf{C})$, we provide an interesting example of a series of nonsymmetric pairs $\left(\mathfrak{g}, \mathfrak{g}_{1}\right)$. It will demonstrate once more that we have to be very careful in the positive characteristic setting; cf. [Š 4 , Sect. 2]. 
Example 2.7. Fix $n \in \mathbb{N}$ and put $\mathfrak{g}=\mathfrak{g}^{n}=\mathfrak{s} l(n, \mathbb{K})$. Define $m_{i}=i(n-i)$, for $1 \leq i<n$, and then $H, E, F \in \mathfrak{g}$ as follows:

$$
H=\sum_{i=1}^{n}(n+1-2 i) E_{i i}, \quad E=\sum_{i=1}^{n-1} m_{i} E_{i, i+1}, \quad F=\sum_{i=1}^{n-1} E_{i+1, i} .
$$

Define a subalgebra $\mathfrak{g}_{1}=\mathfrak{g}_{1}^{n}=\operatorname{span}_{\mathbb{K}}\{H, E, F\}$, and consider a pair $\left(\mathfrak{g}, \mathfrak{g}_{1}\right)$. Now we in particular have $\operatorname{Tr}(E F)=\left(n^{3}-n\right) / 6$. Let $B_{n}=B_{\mathfrak{g}^{n}}$ and $\boldsymbol{\beta}=$ $\boldsymbol{\beta}_{n}=B_{n} \mid \mathfrak{g}_{1}^{n}$. Then for every $n$ such that $p=n+1$ is prime, and $\operatorname{char}(\mathbb{K})=p$, we have $B_{n}$ nondegenerate, while the form $\boldsymbol{\beta}_{n}$ is obviously a degenerate one.

We will finish this section by an easy example which is at the same time very instructive; cf. the proof of Theorem 3.7. It shows what in general the class of pairs $\left(\boldsymbol{G}, \boldsymbol{G}_{1}\right)$ of algebraic $\mathbb{K}$-groups, where $\boldsymbol{G}_{1}$ is self-normalizing in $\boldsymbol{G}$, has to do with the relationship between the adjoint/coadjoint orbits of these groups. To be more precise, take $\left(\mathfrak{g}, \mathfrak{g}_{1}\right)$, a pair of the corresponding Lie algebras. Then we assume that there exists an $\operatorname{Ad} \boldsymbol{G}_{1}$-stable subspace $\mathfrak{p} \leq \mathfrak{g}$ such that: $(\mathrm{R}) \mathfrak{g}=\mathfrak{g}_{1} \oplus \mathfrak{p}$. Then a famous theorem of Richardson ([R]; see also $[\mathrm{Hu}$, Sect. 3.8], [J, Sect. 2]) states that the intersection of any $\boldsymbol{G}$-conjugacy class with $\boldsymbol{G}_{1}$ is a union of finitely many $\boldsymbol{G}_{1}$-conjugacy classes. Analogously, for any $X \in \mathfrak{g}$, the intersection $\operatorname{Ad} \boldsymbol{G}(X) \cap \mathfrak{g}_{1}$ consists of finitely many adjoint $\boldsymbol{G}_{1}$-orbits on $\mathfrak{g}_{1}$. The next reason why we would like to know whether $\boldsymbol{G}_{1}$ is self-normalizing in $\boldsymbol{G}$ is the fact that if this is so, then one can often expect for the mentioned intersections of $\boldsymbol{G}$-classes/orbits to be unions of "small" number of $\boldsymbol{G}_{1}$-classes/orbits.

Example 2.8. Let $G=\operatorname{SL}(2, \mathbb{C})$, and $\mathfrak{g}=\mathfrak{s l}(2, \mathbb{C})$ be its Lie algebra. Define a Cartan subalgebra $\mathfrak{h}=\mathbb{C}\left(\begin{array}{cc}1 & 0 \\ 0 & -1\end{array}\right)$ of $\mathfrak{g}$, and then $H=\mathcal{C}_{G}(\mathfrak{h})$, the centralizer of $\mathfrak{h}$ in $G$. By definition, $H$ is a Cartan subgroup of $G ; H$ consists of all matrices $\left(\begin{array}{cc}a & 0 \\ 0 & a^{-1}\end{array}\right), a \in \mathbb{C}^{\times}$. Now define $N$ to be the normalizer $\mathcal{N}_{G}(H)$. It is easy to see that $N=H \cup\left(\begin{array}{cc}0 & 1 \\ -1 & 0\end{array}\right) H$, and $N$ is a self-normalizing subgroup of $G$; also, as the index $(N: H)=2$, the Lie algebra of $N$ is equal to $\mathfrak{h}$ as well. Let us now compute the adjoint orbits. For that, given any $z \in \mathbb{C}$, define matrices $A_{z, 0}=\left(\begin{array}{cc}z / 2 & 0 \\ 0 & -z / 2\end{array}\right) \in \mathfrak{h}$ and $A_{z, 1}=\left(\begin{array}{cc}z / 2 & 1 \\ 0 & -z / 2\end{array}\right)$. Define orbits $\mathcal{O}_{z, i}^{G}=G . A_{z, i}$, for $i=0,1$, and $\mathcal{O}_{z, 0}^{N}=N \cdot A_{z, 0}$. Then we have

$$
\mathcal{O}_{z, i}^{G} \cap \mathfrak{h}=\left\{ \pm A_{z, 0}\right\}=\mathcal{O}_{z, 0}^{N}, \quad \text { for } i=0,1 ;
$$

note that $H \cdot A_{z, 0}=\left\{A_{z, 0}\right\}$.

\section{Symplectic AND Even orthogonal groups}

Throughout this section we use the notation

$$
\mathfrak{G}=\mathfrak{s l}(2 n, \mathbb{K}) .
$$


3.1. The map $A \mapsto A^{\sharp}$. Suppose that $\mathbb{K}$ has an involution, written as $\alpha \mapsto \bar{\alpha}$; possibly being the identity $1_{\mathbb{K}}$. Let $V$ be a $2 n$-dimensional $\mathbb{K}$-vector space, and fix a basis $\mathcal{B}=\left(e_{1}, \ldots, e_{n}, f_{1}, \ldots, f_{n}\right)$. For $\varepsilon \in\{ \pm 1\}$, define a map $\top=\top_{\varepsilon}: V \rightarrow V, x \mapsto x^{\top}$, given by

$$
\left(\sum_{i} \alpha_{i} e_{i}+\beta_{i} f_{i}\right)^{\top}=\sum_{i} \overline{\alpha_{i}} f_{i}+\varepsilon \overline{\beta_{i}} e_{i}, \quad \text { for } \quad \alpha_{i}, \beta_{i} \in \mathbb{K} .
$$

Let also $\omega=\omega_{\varepsilon}: V \times V \rightarrow \mathbb{K}$ be a bilinear nondegenerate $\varepsilon$-symmetric form; i.e., $\omega_{+}=\omega_{+1}$ is symmetric and $\omega_{-}=\omega_{-1}$ is skew-symmetric. More precisely, we assume that $\omega\left(e_{i}, e_{j}\right)=0=\omega\left(f_{i}, f_{j}\right)$ and $\omega\left(e_{i}, f_{j}\right)=\delta_{i j}$; i.e., for $\varepsilon=1$ (resp. $\varepsilon=-1), \mathcal{B}$ is an orthogonal (resp. symplectic) basis of $V$ with respect to $\omega$.

Define a form $\langle\cdot, \cdot\rangle=\langle\cdot, \cdot\rangle_{\varepsilon}: V \times V \rightarrow \mathbb{K}$,

$$
\langle x, y\rangle=\omega\left(x, y^{\top}\right), \quad \text { for } \quad x, y \in V \text {. }
$$

We clearly have: $(\top 1) x^{\top \top}=\varepsilon x$; and $(\top 2)\left\langle x^{\top}, y\right\rangle=\varepsilon\left\langle y^{\top}, x\right\rangle$. The next three observations we formulate as a lemma.

LEMmA 3.1. (i) The form $\langle\cdot, \cdot\rangle$ is hermitian; i.e., $\overline{\langle x, y\rangle}=\langle y, x\rangle$, for $x, y \in V$.

(ii) $\mathcal{B}$ is an orthogonal basis of $V$ with respect to $\langle\cdot, \cdot\rangle$.

(iii) $\omega(x, y)=\left\langle y, x^{\top}\right\rangle$, for $x, y \in V$.

Now, we have two forms, $\omega$ and $\langle\cdot, \cdot\rangle$, on $V$.

Definition 3.2. For any $A \in$ End $V$ define $A^{\top}: V \rightarrow V$ by $A^{\top}(x)=$ $\left(A\left(x^{\top}\right)\right)^{\top}$, for $x \in V$. Next, define

$$
A^{\sharp}=A_{\varepsilon}^{\sharp}:=\varepsilon\left(A^{*}\right)^{\top} ;
$$

here $A^{*}=A_{\varepsilon}^{*}$ is the $\langle\cdot, \cdot\rangle$-adjoint of $A$, that is, the unique element of End $V$ satisfying $\langle A x, y\rangle=\left\langle x, A^{*} y\right\rangle$ for $x, y \in V$. We will say that $A$ is $\omega$-hermitian (resp. $\omega$-antihermitian) if $A^{\sharp}=A$ (resp. $A^{\sharp}=-A$ ).

The (i) and (ii) of the next lemma were in fact established in [ $\breve{S} 1$, Lemma 2.6]; (iii) is obvious.

LEMmA 3.3. (i) $A^{\sharp}$ is the $\omega$-adjoint of $A$; that is, we have

$$
\omega(A x, y)=\omega\left(x, A^{\sharp} y\right), \quad \text { for } \quad x, y \in V .
$$

(ii) The map $A \mapsto A^{\sharp}$ is a $\mathbb{K}$-linear automorphism of EndV, satisfying $A^{\sharp \sharp}=A$ and $(A B)^{\sharp}=B^{\sharp} A^{\sharp}$, for all $A, B \in$ End $V$.

(iii) For $A \in G L(V)$, we have $\left(A^{-1}\right)^{\sharp}=\left(A^{\sharp}\right)^{-1}$.

Next we need a matrix realization of the map $A \mapsto A^{\sharp}$. For the symplectic case (i.e., $\varepsilon=-1$ ), the following easy lemma was established in [ŠS1, Lemma 3.3]; here $\boldsymbol{A}=A(\mathcal{B})$ and $\boldsymbol{A}^{\sharp}=A^{\sharp}(\mathcal{B})$ are the matrices corresponding to $A$ and $A^{\sharp}$, via $\mathcal{B}$, respectively. For the convenience of the reader we include a short argument. 
Lemma 3.4. For the block-matrices $\boldsymbol{A}$ and $\boldsymbol{A}^{\sharp}$ we have

$$
\boldsymbol{A}^{\sharp}=\left(\begin{array}{cc}
T^{t} & \varepsilon Y^{t} \\
\varepsilon Z^{t} & X^{t}
\end{array}\right), \quad \text { if } \quad \boldsymbol{A}=\left(\begin{array}{cc}
X & Y \\
Z & T
\end{array}\right) ;
$$

here $X, Y, Z, T$ are $n$-by-n matrices.

Proof. Write $X=\left(x_{i j}\right), \ldots, T=\left(t_{i j}\right)$. By Lemma 3.1(ii), $A^{*}(\mathcal{B})=$ $\overline{A(\mathcal{B})}^{t}$. Thus we have

$$
A^{\sharp} e_{j}=\varepsilon\left(A^{*} f_{j}\right)^{\top}=\varepsilon\left(\sum_{i} \overline{z_{j i}} e_{i}+\overline{t_{j i}} f_{i}\right)^{\top}=\sum_{i} t_{j i} e_{i}+\varepsilon z_{j i} f_{i} ;
$$

and, analogously, we compute $A^{\sharp} f_{j}$. Hence the lemma follows.

Similarly as before, for $\varepsilon \in\{ \pm 1\}$ we define a $2 n$-by- $2 n$ matrix $J_{\varepsilon}=J_{+}$or $J_{-}$by

$$
J_{\varepsilon}=\left(\begin{array}{cc}
0 & I \\
\varepsilon I & 0
\end{array}\right)
$$

$J_{+}$(resp. $\left.J_{-}\right)$is called the standard symmetric (resp. skew-symmetric) matrix. Then define a Lie algebra $\mathfrak{G}_{1}^{\varepsilon}$ as the set of all $A \in \mathfrak{G}$ satisfying $J_{\varepsilon} A+A^{t} J_{\varepsilon}=0$; we will write $\mathfrak{G}_{1}^{\varepsilon}=\mathfrak{G}_{1}^{+}$or $\mathfrak{G}_{1}^{-}$. Since $J_{\varepsilon}^{t} A^{t} J_{\varepsilon}=A^{\sharp}$, we have

$$
\mathfrak{G}_{1}^{\varepsilon}=\left\{A \in \mathfrak{G} \mid A^{\sharp}=-A\right\} .
$$

More precisely, as we noted in the Introduction, for a block-matrix $A=\left(\begin{array}{c}X \\ Z\end{array} \underset{T}{Y}\right)$ we have $A \in \mathfrak{G}_{1}^{\varepsilon}$ if and only if

$$
T^{t}+X=0, \quad \varepsilon Y^{t}+Y=0, \quad \varepsilon Z^{t}+Z=0 .
$$

Notice that, by Lemma 3.3 , the map

$$
\theta=\theta^{\varepsilon}: A \mapsto-A_{\varepsilon}^{\sharp}
$$

is an involution on $\mathfrak{G}$; for $\varepsilon=1$ (resp. $\varepsilon=-1$ ) we call it the orthogonal (resp. symplectic) involution. Thus $\left(\mathfrak{G}, \mathfrak{G}_{1}^{\varepsilon}\right)$ is a symmetric pair. Then define $\mathfrak{P}^{\varepsilon}$ to be the subspace of $\mathfrak{G}$ consisting of $\omega$-hermitian matrices; i.e.,

$$
\mathfrak{P}^{\varepsilon}=\left\{A \in \mathfrak{G} \mid A^{\sharp}=A\right\} .
$$

Of course, by Proposition 2.1, we have a direct sum decomposition $\mathfrak{G}=$ $\mathfrak{G}_{1}^{\varepsilon} \oplus \mathfrak{P}^{\varepsilon}$.

3.2. On normalizers for Lie algebras. In this subsection, keeping the notation of the previous one, we state a proposition which is a technical result providing some useful information concerning certain normalizers and centralizers. First an easy lemma.

Lemma 3.5. Let $n>1$ and suppose $C \in M_{n}(\mathbb{K})$ is a matrix such that $[X, C]=0$ for every $X \in M_{n}(\mathbb{K})$ satisfying $\varepsilon X^{t}+X=0$. For $n \geq 3$ or $\varepsilon=-1, C$ is a scalar matrix; for $n=2$ and $\varepsilon=1, C$ is of the form $\left(\begin{array}{cc}c_{1} & c_{2} \\ -c_{2} & c_{1}\end{array}\right)$, where $c_{1}, c_{2} \in \mathbb{K}$. 
Proof. We consider the case $n \geq 3$; the rest is clear. Let $C=\left(c_{i j}\right)$, and fix a pair of indices $(i, j), i<j$. We will show that $c_{i j}=0$. For that purpose define a matrix $X=E_{k l}-\varepsilon E_{l k}$. We have

$$
0=[X, C]=\sum_{a=1}^{n}\left(c_{l a} E_{k a}-c_{a k} E_{a l}\right)-\varepsilon\left(c_{k a} E_{l a}-c_{a l} E_{a k}\right) .
$$

Choose $k=j$ and $l \neq i, j$. Note that the coefficient by $E_{i l}$ in the above expression is equal to $c_{i j}$; i.e., $c_{i j}=0$. Next note that the coefficient by $E_{k l}$ equals $c_{l l}-c_{k k}$. Thus, $C=\alpha I$, for $\alpha=c_{11}=\cdots=c_{n n}$.

Proposition 3.6. Suppose that either $\operatorname{char}(\mathbb{K})=0$ or $\operatorname{char}(\mathbb{K})=p$ and $p$ does not divide $n$.

(i) The Lie algebra $\mathfrak{G}_{1}^{\varepsilon}$ is self-normalizing in $\mathfrak{G}$.

(ii) The centralizer $C_{\mathfrak{G}}\left(\mathfrak{G}_{1}^{\varepsilon}\right)$ is equal to zero if either $n>1$ or $n=1$ and $\varepsilon=-1 ;$ for $n=1, C_{\mathfrak{G}}\left(\mathfrak{G}_{1}^{+}\right)=\mathfrak{G}_{1}^{+}$.

(iii) The normalizer $N_{\mathfrak{G}}\left(\mathfrak{P}^{\varepsilon}\right)$ is equal to $\mathfrak{G}_{1}^{\varepsilon}$.

Proof. We will treat the case $n>1$; note that for $n=1$ we have $\mathfrak{G}_{1}^{-}=\mathfrak{G}$ and $\mathfrak{G}_{1}^{+}=\mathbb{K}\left(\begin{array}{cc}1 & 0 \\ 0 & -1\end{array}\right)$.

Write $\mathfrak{G}_{1}, \mathfrak{P}$ and $J$ instead of $\mathfrak{G}_{1}^{\varepsilon}, \mathfrak{P}^{\varepsilon}$ and $J_{\varepsilon}$.

(i) Let $M \in \mathfrak{G}$ be such that $B=[M, A] \in \mathfrak{G}_{1}$, for all $A \in \mathfrak{G}_{1}$. Then $J B+B^{t} J=0$, which is further equivalent to

$$
A^{t}\left(J M+M^{t} J\right)+\left(J M+M^{t} J\right) A=0 .
$$

Write $A=\left(\begin{array}{cc}\underset{Z}{Z} & T\end{array}\right)$, as before, and $M=\left(\begin{array}{ll}N & P \\ Q & R\end{array}\right)$. By putting $T=-X^{t}$ and $Y=Z=0$ in (3.2), we obtain that

$$
\begin{array}{r}
X^{t}\left(Q+\varepsilon Q^{t}\right)+\left(Q+\varepsilon Q^{t}\right) X=0, \\
X\left(\varepsilon P+P^{t}\right)+\left(\varepsilon P+P^{t}\right) X^{t}=0, \\
\left(N+R^{t}\right) X=X\left(N+R^{t}\right),
\end{array}
$$

for all $X \in M_{n}(\mathbb{K})$. By choosing $X=I$, it follows that both $Q+\varepsilon Q^{t}=0$ and $P+\varepsilon P^{t}=0$. Further, from the last equality above we have that $N+R^{t}$ is a scalar matrix; i.e., $N+R^{t}=k I$, for some $k \in \mathbb{K}$. Since the trace $0=\operatorname{Tr} M=\operatorname{Tr}\left(N+R^{t}\right)=n k$, it follows that $k=0$; here we use that $p$ does not divide $n$. That is, $N+R^{t}=0$. Hence, $M \in \mathfrak{G}_{1}$; cf. (3.1).

(ii) Let $M \in \mathfrak{G}$ be such that $[M, A]=0$, for all $A \in \mathfrak{G}_{1}$. Write $A$ and $M$ as in (i), and put, again, $T=-X^{t}$ and $Y=Z=0$. Then it immediately follows that $P=Q=0$, while both $N$ and $R$ are scalar matrices, i.e., $N=k I$ and $R=l I$, for some $k, l \in \mathbb{K}$. Choose now $X=Z=T=0$, and $Y$ non-zero satisfying $\varepsilon Y^{t}+Y=0$. Then $M A=A M$ implies $k Y=l Y$, and hence $k=l$. Again, the trace argument gives that $k=l=0$; and here we again use that $p$ does not divide $n$. Thus $M=0$, as we claimed. 
(iii) Let $M \in \mathfrak{G}$ be such that $[M, A] \in \mathfrak{P}$, for all $A \in \mathfrak{P}$. By denoting $B=M+M^{\sharp},[M, A] \in \mathfrak{P}$ is equivalent to $[A, B]=0$. Write $M$ as above, and choose $A=\left(\begin{array}{cc}X & 0 \\ 0 & X^{t}\end{array}\right)$. It is easy to see that then $\left[X, N+R^{t}\right]=0$ and

$$
X\left(P+\varepsilon P^{t}\right)=\left(P+\varepsilon P^{t}\right) X^{t}, \quad X^{t}\left(Q+\varepsilon Q^{t}\right)=\left(Q+\varepsilon Q^{t}\right) X .
$$

Since $X$ is arbitrary, we clearly have $N+R^{t}=0$. At the same time, if we put $C=P+\varepsilon P^{t}$, then the first of the above two equalities reads as $X C=C X^{t}$. By Lemma 3.5, it is obvious that $C=0$. Analogously, $Q+\varepsilon Q^{t}=0$. We conclude that $M \in \mathfrak{G}_{1}$.

3.3. On normalizers for groups. We have defined the Lie algebras $\mathfrak{G}_{1}^{\varepsilon}$. Also, for $\omega=\omega_{+}$or $\omega_{-}$, we define a group

$$
\mathcal{G}_{1}(\omega)=\{g \in \mathrm{SL}(V) \mid \omega(g x, g y)=\omega(x, y) \quad \text { for all } x, y \in V\} .
$$

Then, by what we had in Subsections 1.2 and 3.1,

$$
\mathcal{G}_{1}(\omega) \cong G_{1}(\omega)=\left\{A \in \mathrm{SL}(2 n, \mathbb{K}) \mid A A^{\sharp}=I\right\} .
$$

Since $\omega_{+}$is symmetric and $\omega_{-}$is skew-symmetric,

$$
G_{1}^{+}=G_{1}\left(\omega_{+}\right)=\operatorname{SO}(2 n, \mathbb{K}) \quad \text { and } \quad G_{1}^{-}=G_{1}\left(\omega_{-}\right)=\operatorname{Sp}(2 n, \mathbb{K})
$$

(note that our notation here is in fact slightly imprecise, as the SO- and Spgroups realizations depend strictly on the chosen involution $\theta^{\varepsilon}$, i.e., the map $\left.A \mapsto A_{\varepsilon}^{\sharp}\right)$.

Let us now state our main result. For that, consider a pair of groups $G_{1} \subseteq G$,

$$
\left(G, G_{1}\right)=\left(\mathrm{SL}(2 n, \mathbb{K}), G_{1}(\omega)\right) .
$$

Theorem 3.7. (i) Suppose that $n>1$ or $\varepsilon=1$. Then we have

$$
\mathcal{N}_{G}\left(G_{1}\right)=\left\{M \in G \mid M^{\sharp} M=\lambda I, \quad \lambda \in \boldsymbol{\mu}_{2 n}(\mathbb{K})\right\} .
$$

Furthermore, for a certain group epimorphism $\sigma$ we have a short exact sequence of groups

$$
1 \rightarrow G_{1} \hookrightarrow \mathcal{N}_{G}\left(G_{1}\right) \stackrel{\sigma}{\rightarrow} \boldsymbol{\mu}_{k}(\mathbb{K}) \rightarrow 1 ;
$$

here $k=2 n$ if the Condition $(\diamond)$ holds, and $k=n$ if not.

For $n=1$ we have $\mathcal{N}_{G}\left(G_{1}^{-}\right)=G_{1}^{-}=G$.

(ii) If $n>1$ or $\varepsilon=-1$, then the normalizer $\mathcal{N}_{G}\left(G_{1}\right)$ is self-normalizing in $G$. If $n=\varepsilon=1$, then $\mathcal{N}_{G}\left(G_{1}\right)$ is self-normalizing in $G$ if and only if $\mathbb{K}$ is different than $\mathbb{F}_{3}$ or $\mathbb{F}_{5}$.

Proof. (i) Let $M \in \mathcal{N}_{G}\left(G_{1}\right)$. Then $B=M A M^{-1} \in G_{1}$, for all $A \in G_{1}$. Thus we have $I=B^{\sharp} B$, and hence, using Lemma 3.3, it immediately follows that

$$
M^{\sharp} M \in \mathcal{C}_{G}\left(G_{1}\right) .
$$


Let us first consider the case $n=1$ and $\varepsilon=1$; here we will obtain a precise description of $\mathcal{N}_{G}\left(G_{1}\right)$. We have

$$
G_{1}=\left\{\left(\begin{array}{cc}
a & 0 \\
0 & a^{-1}
\end{array}\right) \mid a \in \mathbb{K}^{\times}\right\},
$$

and it is also easy to check that $\mathcal{C}_{G}\left(G_{1}\right)=G_{1}$. Then the above condition on $M$ reads as $M^{\sharp} M=\left(\begin{array}{cc}a & 0 \\ 0 & a^{-1}\end{array}\right)$, for some $a \in \mathbb{K}^{\times}$, which implies that $a^{2}=1$. Thus the description of $\mathcal{N}_{G}\left(G_{1}\right)$, as stated in the theorem, holds. More precisely, we have a (disjoint) union

$$
\mathcal{N}_{G}\left(G_{1}\right)=G_{1} \cup\left\{\left(\begin{array}{cc}
0 & y \\
-y^{-1} & 0
\end{array}\right) \mid y \in \mathbb{K}^{\times}\right\} ;
$$

cf. Examples 2.8 and 4.7. Now, for the first part of (i) it remains to take into account the following claim. Although it is probably well known, for the convenience of the reader and later needs we provide a proof.

Claim 1. If $n>1$, then the centralizer

$$
\mathcal{C}_{G}\left(G_{1}\right)=\left\{\lambda I \mid \lambda \in \boldsymbol{\mu}_{2 n}(\mathbb{K})\right\} .
$$

Proof. Let $M \in G$ be such that $A M=M A$, for all $A \in G_{1}$; and write it as

$$
M=\left(\begin{array}{ll}
N & P \\
Q & R
\end{array}\right) .
$$

Take in particular

$$
A=\left(\begin{array}{cc}
I & Y \\
0 & I
\end{array}\right) \in G_{1},
$$

and note that then $\varepsilon Y^{t}+Y=0$. It is straightforward to see that $A M=M A$ is equivalent to the equalities

$$
Y Q=Q Y=0 \quad \text { and } \quad Y R=N Y .
$$

We will treat the following three cases separately:

(S) Symplectic case (i.e., $\varepsilon=-1$ );

(O1) Orthogonal case (i.e., $\varepsilon=1$ ), and $n$ odd;

(O2) Orthogonal case, and $n$ even.

For $(\mathbf{S})$ we take $Y=I$, and thus both $Q=0$ and $R=N$. Analogously, $P=0$, and therefore $M=\left(\begin{array}{cc}N & 0 \\ 0 & N\end{array}\right)$. Take now $A=\left(\begin{array}{cc}X & 0 \\ 0 & \left(X^{-1}\right)^{t}\end{array}\right)$. Then it immediately follows that $M=\lambda I$, where $\lambda^{2 n}=1$.

For (O2) we take $Y=S_{r,-}$, where the later matrix is given by (1.1) and $r=n / 2$. As $Y$ is regular, we have that $Q=0$; and, analogously, $P=0$. The rest of the argument is the same as for $(\mathbf{S})$. 
For $(\mathbf{O 1})$ we take $Y$ to be the block-matrix

$$
Y=\left(\begin{array}{ccc}
0 & 0 & \boldsymbol{s}_{r-1} \\
0 & 0 & 0 \\
-\boldsymbol{s}_{r-1} & 0 & 0
\end{array}\right)
$$

where $2 r=n+1$. Since $Y Q=Q Y$, we deduce that $Q=q E_{r r}$, for some $q \in \mathbb{K}$. Take, again, $A=\left(\begin{array}{cc}X & 0 \\ 0 & \left(X^{-1}\right)^{t}\end{array}\right)$, and assume that moreover in the matrix $X=\left(x_{i j}\right)$ we have $x_{r r}^{2} \neq 1$. As $M A=A M$ implies in particular that $X^{t} Q X=Q$, it is straightforward to conclude that $q=0$. Now, as before, we deduce that $M=\lambda I, \lambda^{2 n}=1$. subset

Let us now show the second part of (i). For any $\lambda \in \boldsymbol{\mu}_{2 n}(\mathbb{K})$ define a

$$
\Gamma_{\lambda}=\left\{M \in G \mid M^{\sharp} M=\lambda I\right\}
$$

of $G$; note that $\Gamma_{1}=G_{1}$. Next, for $\lambda \in \boldsymbol{\mu}_{n}(\mathbb{K})$, define a matrix $M_{\lambda}=\left(\begin{array}{cc}\lambda I & 0 \\ 0 & I\end{array}\right)$. Obviously, $M_{\lambda} \in \Gamma_{\lambda}$, and so in particular $\Gamma_{\lambda} \neq \emptyset$. For what follows we need this claim.

Claim 2. The Condition $(\diamond)$ is further equivalent to the following one: For every $\lambda \in \boldsymbol{\mu}_{2 n}(\mathbb{K})$, we have $\Gamma_{\lambda} \neq \emptyset$.

Proof. $(\diamond)$. It is easy to check that the map

$$
d: \boldsymbol{\mu}_{n}(\mathbb{K}) \rightarrow \boldsymbol{\mu}_{2 n}(\mathbb{K}) \backslash \boldsymbol{\mu}_{n}(\mathbb{K}), \quad d(\lambda)=\omega \lambda,
$$

is bijective. Next, we obviously have $W M_{\lambda} \in \Gamma_{d(\lambda)}$, which proves the claim.

Suppose now that the Condition $(\diamond)$ holds; an argument for the opposite is completely analogous. Then, for any $\lambda \in \boldsymbol{\mu}_{2 n}(\mathbb{K})$ and $U_{\lambda} \in \Gamma_{\lambda}$, the map $G_{1} \ni g \mapsto U_{\lambda} g \in \Gamma_{\lambda}$ is bijective. Thus we have a disjoint union, into nonempty sets,

$$
\mathcal{N}_{G}\left(G_{1}\right)=\bigcup_{\lambda \in \mu_{2 n}(\mathbb{K})} \Gamma_{\lambda} .
$$

Since $\Gamma_{\lambda_{1}} \Gamma_{\lambda_{2}} \subseteq \Gamma_{\lambda_{1} \lambda_{2}}$, it is clear that the map

$$
\sigma: \mathcal{N}_{G}\left(G_{1}\right) \rightarrow \boldsymbol{\mu}_{2 n}(\mathbb{K}), \quad \sigma_{\mid \Gamma_{\lambda}}=\lambda,
$$

is a group epimorphism with the kernel $\operatorname{Ker} \sigma=G_{1}$. This finishes the proof of (i).

(ii) Loosely speaking, our proof starts similarly as the one of Claim 1. But, as we will see, our argument here will be significantly refined.

Write $\mathrm{N}=\mathcal{N}_{G}\left(G_{1}\right)$, and let $D \in \mathcal{N}_{G}(\mathrm{~N})$. Then, in particular,

$$
X_{A}=D A D^{-1} \in \mathrm{N}, \quad \text { for } A \in G_{1} .
$$


Put $M=D^{\sharp} D$. Obviously, in order to prove that $D \in \mathrm{N}$ it is sufficient to show that $M \in \mathcal{C}_{G}\left(G_{1}\right)$. Now, analogously as in (i), $X_{A} \in \mathrm{N}$ if and only

$$
[M, A]=\lambda_{A} I, \quad \text { for some } \lambda_{A} \in \boldsymbol{\mu}_{2 n}(\mathbb{K}) .
$$

Write now $M$ as in (3.5), and let $A$ be any matrix as in (3.6). It is immediate that for any such $A$ satisfying (3.9) we necessarily have $\lambda_{A}=1$; more precisely, $\lambda_{A} \neq 1$ would imply $M=0$, which is impossible. In other words, for any such $A$ the equalities (3.7) hold.

Suppose now we have the case $(\mathbf{S})$ of Claim 1 , where $n>1$. As there we deduce that again $M=\left(\begin{array}{cc}N & 0 \\ 0 & N\end{array}\right)$, and also $Y N=N Y$ for any $Y$ satisfying $Y^{t}=Y$. Take first $Y=\left(y_{i j}\right)$ to be a diagonal matrix satisfying $y_{i i} \neq y_{j j}$ for particular $i \neq j$. If we put $N=\left(\nu_{i j}\right)$, then on the $(i, j)$-th place of $N Y$ we have $\nu_{i j} y_{j j}$, while on the $(i, j)$-th place of $Y N$ we have $y_{i i} \nu_{i j}$. Hence, necessarily $\nu_{i j}=0$. As $i \neq j$ were arbitrary, we conclude that $N$ is diagonal. Take now $Y=E_{i j}+E_{j i}$, where $i \neq j$. By the equality $N Y=Y N$ we deduce that $\nu_{i i}=\nu_{j j}$. That is, $M=\lambda I$ for some $\lambda \in \boldsymbol{\mu}_{2 n}(\mathbb{K})$, as we had to see.

Let us now consider the case (O2). As in Claim 1, we conclude that $M=\left(\begin{array}{cc}N & 0 \\ 0 & R\end{array}\right)$, where $Y R=N Y$ for any $Y$ satisfying $Y^{t}+Y=0$. Write again $N=\left(\nu_{i j}\right)$, and $R=\left(\rho_{i j}\right)$. Take

$$
Y=E_{i j}-E_{j i}, \quad \text { where } i \neq j .
$$

Furthermore, suppose that $n \geq 3$. Then on the $(i, j)$-th place of $N Y$ we have $\nu_{i i}$, while on the $(i, j)$-th place of $Y R$ we have $\rho_{j j}$. Thus we deduce that $\nu_{i i}=\rho_{j j}$, for $i \neq j$. Hence, in particular, $\nu_{11}=\rho_{j j}$ for any $j \geq 2$; and at the same time $\rho_{11}=\nu_{22}=\rho_{33}$. Therefore, $\rho_{11}=\cdots=\rho_{n n}$, and analogously

$$
\nu_{11}=\cdots=\nu_{n n}=\rho_{11}=\cdots=\rho_{n n} .
$$

Fix now an arbitrary pair of indices $(u, v)$, where $u \neq v$. We may assume for $Y$ as in (3.10) the following: $i=v$ and $j \neq u, v$. Then, by considering the coefficients by the matrix $E_{u j}$ in the equality $N Y=Y R$, we deduce that $\nu_{u v}=0$. Analogously, $\rho_{u v}=0$. Finally, by the later and (3.11) we obtain that again $M=\lambda I$ for some $\lambda \in \boldsymbol{\mu}_{2 n}(\mathbb{K})$.

Suppose the case $(\mathbf{O 1})$ holds. Take first $Y$ to be as in (3.8). As there we deduce that $Q=q E_{r r}, q \in \mathbb{K}$. Take then $Y=E_{r, r+1}-E_{r+1, r}$. By the equality $Q Y=0$, we have $q=0$, i.e., $Q=0$. Analogously, $P=0$. That is, $M=\left(\begin{array}{cc}N & 0 \\ 0 & R\end{array}\right)$. Now, the same argument as for $(\mathbf{O 2})$ works here again.

What remains to do is to treat three cases when $n \leq 2$. Consider first the case when $\varepsilon=-1$ and $n=1$. Then, as we have showed, $\mathrm{N}=G$. Hence, $\mathcal{N}_{G}(\mathrm{~N})=\mathrm{N}$.

Consider now the case $\varepsilon=1$ and $n=1$. Then $G_{1}$ and $\mathrm{N}$ are given by (3.3) and (3.4), respectively. Let us show that again $\mathcal{N}_{G}(\mathrm{~N})=\mathrm{N}$, provided that $\mathbb{K}$ is not $\mathbb{F}_{3}$ or $\mathbb{F}_{5}$. For that purpose take $D=\left(\begin{array}{cc}x & y \\ z & t\end{array}\right) \in \mathcal{N}_{G}(\mathrm{~N})$. Then choose 
some $a_{0} \in \mathbb{K}^{\times}$satisfying $a_{0}^{2} \neq \pm 1$, and put $A_{0}=\left(\begin{array}{cc}a_{0} & 0 \\ 0 & a_{0}^{-1}\end{array}\right) \in G_{1}$. Define

$$
D A_{0} D^{-1}=\left(\begin{array}{cc}
a_{0}+y z\left(a_{0}-a_{0}^{-1}\right) & -x y\left(a_{0}-a_{0}^{-1}\right) \\
z t\left(a_{0}-a_{0}^{-1}\right) & a_{0}-x t\left(a_{0}-a_{0}^{-1}\right)
\end{array}\right) \in \mathrm{N} .
$$

If we would have $x y \neq 0$, then $a_{0}^{2} \neq 1$ and (3.4) would imply that

$$
a_{0}+y z\left(a_{0}-a_{0}^{-1}\right)=a_{0}-x t\left(a_{0}-a_{0}^{-1}\right)=0 .
$$

Summing up the later two equalities we obtain that $a_{0}^{2}=-1$; a contradiction. Thus, $x y=0$. Analogously, $z t=0$. An easy discussion shows that the only two possibilities are: $x=t=0$ and $y, z \neq 0$, or $y=z=0$ and $x, t \neq 0$; i.e., $D=\left(\begin{array}{ll}0 & y \\ z & 0\end{array}\right)$ or $D=\left(\begin{array}{ll}x & 0 \\ 0 & t\end{array}\right)$. But this means that necessarily $D \in \mathrm{N}$; and we are done. We leave to the reader to check that for $\mathbb{K}=\mathbb{F}_{3}$ or $\mathbb{F}_{5}$ we have $\mathcal{N}_{G}(\mathrm{~N}) \neq \mathrm{N}$.

The last case is $\varepsilon=1$ and $n=2$. As before, we again have $M=\left(\begin{array}{cc}N & 0 \\ 0 & R\end{array}\right)$, where $Y R=N Y$ for any $Y$ satisfying $Y^{t}+Y=0$. But the only such one is $Y=\left(\begin{array}{cc}0 & 1 \\ -1 & 0\end{array}\right)$. Hence it follows that for $N=\left(\begin{array}{ll}\nu_{11} & \nu_{12} \\ \nu_{21} & \nu_{22}\end{array}\right)$ we have $R=\left(\begin{array}{cc}\nu_{22} & -\nu_{21} \\ -\nu_{12} & \nu_{11}\end{array}\right)$. Now we will take $A=\left(\begin{array}{cc}X & 0 \\ 0 & \left(X^{-1}\right)^{t}\end{array}\right) \in G_{1}$, where $X=\left(\begin{array}{ll}1 & x \\ 0 & 1\end{array}\right)$. Then, for this $A$, we have (3.9). An easy inspection shows that again necessarily $\lambda_{A}=1$. In other words, $M A=A M$. By choosing $x \neq 0$ it follows at once that $\nu_{12}=\nu_{21}=0$ and $\nu_{11}=\nu_{22}$; i.e., $M=\lambda I$, where $\lambda \in \boldsymbol{\mu}_{4}(\mathbb{K})$.

This finishes the proof of (ii), and so the proof of our theorem.

Concerning the structure of $\mathrm{N}=\mathcal{N}_{G}\left(G_{1}\right)$, the following is an improvement of the above.

Corollary 3.8. The set $\mathrm{N}^{\prime}=\left\{M \in G \mid M^{\sharp} M=\lambda I, \lambda \in \boldsymbol{\mu}_{n}(\mathbb{K})\right\}$ is a normal subgroup of $\mathrm{N}$, and for it we have $\mathrm{N}^{\prime} \cong G_{1} \rtimes \mu_{n}(\mathbb{K})$. Furthermore, $\mathrm{N} / \mathrm{N}^{\prime} \cong \mathbb{Z} / 2 \mathbb{Z}$ if Condition $(\diamond)$ holds, and $\mathrm{N}=\mathrm{N}^{\prime}$ if not.

Proof. With $M_{\lambda}$ as before, define $\mathrm{F}=\left\{M_{\lambda} \mid \lambda \in \boldsymbol{\mu}_{n}(\mathbb{K})\right\}$. Then $\mathrm{F}$ is a finite subgroup of $\mathrm{N}, \mathrm{F} \cong \boldsymbol{\mu}_{n}(\mathbb{K})$ and $\mathrm{F} \cap G_{1}=\{I\}$. As we have a (disjoint) union $\mathrm{N}^{\prime}=\bigcup_{\lambda \in \boldsymbol{\mu}_{n}(\mathbb{K})} \Gamma_{\lambda}$, it is clear that $G_{1} \mathrm{~F}=\mathrm{N}^{\prime}$. Thus we conclude that $\mathrm{N}^{\prime} \cong G_{1} \rtimes \mathrm{F}$. Now, if Condition $(\diamond)$ does not hold, then $\mathrm{N}=\mathrm{N}^{\prime}$, and we are done.

Suppose next that Condition $(\diamond)$ holds, and let $\omega$ and $W$ be as in it. Take any $X \in \mathrm{N} \backslash \mathrm{N}^{\prime}$. Then $X^{\sharp} X=\lambda I$, with $\lambda \in \boldsymbol{\mu}_{2 n}(\mathbb{K}) \backslash \boldsymbol{\mu}_{n}(\mathbb{K})$. Hence, for $Y=W^{-1} X$, we have $Y^{\sharp} Y=\lambda \omega^{-1} I$. We conclude that $Y \in \mathrm{N}^{\prime}$, and so $X \in W \mathrm{~N}^{\prime}$. Thus

$$
\mathrm{N}=\mathrm{N}^{\prime} \cup W \mathrm{~N}^{\prime},
$$

a disjoint union. Define now a map $\tau: \mathrm{N} \rightarrow \mathbb{Z} / 2 \mathbb{Z}, \tau_{\mid \mathrm{N}^{\prime}}=\overline{0}$ and $\tau_{\mid W \mathrm{~N}^{\prime}}=\overline{1}$; cf. Example 4.7, again. Take any $Y_{1}, Y_{2} \in \mathrm{N}^{\prime}$. Using the facts $W \mathrm{~N}^{\prime}=\mathrm{N}^{\prime} W$ and $W^{2} \in \mathrm{N}^{\prime}$, it immediately follows that $Y_{1}\left(W Y_{2}\right) \in W \mathrm{~N}^{\prime}$ and $\left(W Y_{1}\right)\left(W Y_{2}\right) \in \mathrm{N}^{\prime}$. This shows that $\tau$ is a group epimorphism with the kernel $\mathrm{N}^{\prime}$, and so we have our corollary proved. 
Concerning the Condition $(\diamond)$ we note the following easy observation. Let us emphasize that we do not know what happens in general for $\varepsilon=1$ and $n$ even.

Lemma 3.9. Suppose that $\varepsilon=1$ and $n$ is odd. Then for any $\mathbb{K}$, the Condition $(\diamond)$ holds.

Proof. Let $\lambda \in \boldsymbol{\mu}_{2 n}(\mathbb{K})$ be arbitrary. Let $M_{\lambda}=\left(\begin{array}{cc}\lambda I & 0 \\ 0 & I\end{array}\right)$, as before, and $N_{\lambda}=\left(\begin{array}{cc}0 & \lambda I \\ I & 0\end{array}\right)$. As we have $\operatorname{det} M_{\lambda}=\lambda^{n}$ and $\operatorname{det} N_{\lambda}=-\lambda^{n}$, for every odd $n$, it follows that either $M_{\lambda} \in G$ or $N_{\lambda} \in G$. It only remains to note that $M_{\lambda}^{\sharp} M_{\lambda}=\lambda I=N_{\lambda}^{\sharp} N_{\lambda}$.

Compared to the previous one, the next lemma is more complicated. Let us emphasize that our argument strongly relies on the notion of $J$-twisted Pfaffian, introduced in [Š2]; it is a natural counterpart of the standard Pfaffian while working in the symplectic setting.

Lemma 3.10. Suppose that $\varepsilon=-1$ and $n \equiv 2,3(\bmod 4)$. Then, for $\mathbb{K}=\mathbb{C}$, the Condition $(\diamond)$ does not hold.

Proof. Let $J=J_{-}$. Also, define $\omega-\mathcal{H}=\mathfrak{P}^{-} \oplus \mathbb{C} I$, the set of all $\omega$ hermitian complex matrices of size $2 n$; cf. Definition 3.2. For any $X \in \omega-\mathcal{H}$, define the $J$-twisted Pfaffian of $X$ as

$$
J-\operatorname{Pf}(X)=\operatorname{Pf}(J X) ;
$$

here $\operatorname{Pf}$ is the standard Pfaffian, taken so that $\operatorname{Pf}(J)=-1$. Now, for $c \in \mathbb{C}$ and $n>1$, define scalar matrices $A_{n}=c I \in M_{2 n}(\mathbb{C})$, and $X_{n}=J A_{n}=\left(\begin{array}{cc}0 & c I \\ -c I & 0\end{array}\right)$. Next, recall a well known expansion for inductive computation of the Pfaffian (see [A, Ch. III, §5]): For $M=\left(m_{i j}\right) \in M_{2 n}(\mathbb{C})$,

$$
\operatorname{Pf}(M)=\sum_{t=2}^{2 n}(-1)^{t} m_{1 t} \operatorname{Pf}\left(\widehat{M}_{1 t}\right),
$$

where $\widehat{M}_{1 t} \in M_{2 n-2}(\mathbb{C})$ is obtained by deleting both the 1 -st and $t$-th row and column in $M$. Hence it follows that

$$
J-\operatorname{Pf}\left(A_{n}\right)=(-1)^{n+1} c J-\operatorname{Pf}\left(A_{n-1}\right) .
$$

Since in particular $J-\operatorname{Pf}\left(A_{2}\right)=-c^{2}$ (cf. [Š2, Lemma 2.1]), we have proved the following

$$
\text { Claim. } J-\operatorname{Pf}\left(A_{n}\right)= \begin{cases}c^{n} & \text { if } n \equiv 0,1(\bmod 4), \\ -c^{n} & \text { if } n \equiv 2,3(\bmod 4) .\end{cases}
$$

Suppose now that we have some $\omega \in \boldsymbol{\mu}_{2 n}(\mathbb{C}) \backslash \boldsymbol{\mu}_{n}(\mathbb{C})$ and $W \in G=$ $\mathrm{SL}(2 n, \mathbb{C})$ such that $W^{\sharp} W=\omega I$. By the above Claim, $J-\operatorname{Pf}(\omega I)=1$. On the other hand, a nontrivial observation in [Š2, Prop. 1.6] states that $J$-Pf $\left(M^{\sharp} M\right)=-1$ for all $M \in G$, yielding a contradiction. 
REMARK 3.11. The argument given in [Š2] which proves the mentioned Proposition 1.6 is of an analytic flavor. As usual, one can expect to have the same result for an arbitrary algebraically closed field $\mathbb{K}$ of characteristic zero. It would be good to find out if this is so. In particular then we would have for such $\mathbb{K}$ the previous lemma as well.

\section{Remarks on various embeddings of $G_{1}$ into $G$}

Concerning the problem of normalizers, it is interesting to consider various embeddings of Lie algebras $\mathfrak{g}_{1} \hookrightarrow \mathfrak{g}$, and groups $G_{1} \hookrightarrow G$; see Remark 4.4. Next we want to find out which of them are mutually conjugate; cf. Lemmas 4.3 and 4.5. Namely, suppose for the moment that $\mathfrak{g}=\mathfrak{s l}(n, \mathbb{K}), G=\operatorname{SL}(n, \mathbb{K})$ and $\mathfrak{h}$ is a Lie algebra for which we have two faithful representations $\rho_{1,2}$ : $\mathfrak{h} \rightarrow \mathfrak{g}$. Denote $\mathfrak{s}_{i}=\rho_{i}(\mathfrak{h})$; we say that $\mathfrak{s}_{i} \hookrightarrow \mathfrak{g}$ are two embeddings of $\mathfrak{h}$ in $\mathfrak{g}$ realized via $\rho_{i}$, for $i=1,2$. Now, it is possible that the normalizers of $\mathfrak{s}_{i}$ in $\mathfrak{g}$ are not isomorphic; such $\mathfrak{s}_{1}$ and $\mathfrak{s}_{2}$ cannot be $\mathrm{GL}(n, \mathbb{K})$-conjugate. The same phenomenon can happen for some subgroups $S_{1}, S_{2} \leq G$ as well. Related to what we said, the following obvious lemma contains a useful observation; of course, an analogous result holds for Lie algebras as well.

Lemma 4.1. Let $G$ be any group. Suppose that $S_{1}, S_{2} \leq G$ are conjugate subgroups. Then the normalizers $\mathcal{N}_{G}\left(S_{1}\right)$ and $\mathcal{N}_{G}\left(S_{2}\right)$ are conjugate subgroups too. As a consequence, $S_{1}$ is self-normalizing in $G$ if and only if $S_{2}$ is the same.

Now we will consider another realizations of $\mathfrak{s p}(2 n, \mathbb{K})$ and $\mathfrak{s o}(2 n, \mathbb{K})$, the symplectic and even orthogonal Lie algebra of matrices of size $2 n$, in $\mathfrak{G}=$ $\mathfrak{s l}(2 n, \mathbb{K})$. We will also consider realizations of the corresponding, symplectic and orthogonal, groups. But first recall two standard realizations, and some well known related facts; see [B2, Ch. VIII, §13] or [GW, Sect. 1.2.2].

Suppose for the moment that $\mathbb{K}=\overline{\mathbb{K}}$. Let $\mathcal{V}$ be a finite-dimensional $\mathbb{K}$ vector space, and let $\varphi: \mathcal{V} \times \mathcal{V} \rightarrow \mathbb{K}$ be a bilinear nondegenerate form which is either symmetric or skew-symmetric. We define a Lie algebra $\mathfrak{g}(\varphi) \leq \mathfrak{g l}(\mathcal{V})$ and an algebraic group $\boldsymbol{G}(\varphi) \leq \mathrm{GL}(\mathcal{V})$ as follows:

$$
\begin{aligned}
\mathfrak{g}(\varphi) & =\{X \in \mathfrak{g l}(\mathcal{V}) \mid \varphi(X v, W)+\varphi(v, X w)=0 \text { for all } v, w \in \mathcal{V}\}, \\
\boldsymbol{G}(\varphi) & =\{g \in \operatorname{GL}(\mathcal{V}) \mid \varphi(g v, g w)=\varphi(v, w) \text { for all } v, w \in \mathcal{V}\}
\end{aligned}
$$

Suppose $\varphi^{\prime}$ is another bilinear nondegenerate form which is also symmetric/ skew-symmetric if $\varphi$ is the same. Define $\mathfrak{g}\left(\varphi^{\prime}\right)$ and $\boldsymbol{G}\left(\varphi^{\prime}\right)$ as for $\varphi$. Then $\mathfrak{g}\left(\varphi^{\prime}\right) \cong \mathfrak{g}(\varphi)$, an isomorphism of Lie algebras; and $\boldsymbol{G}\left(\varphi^{\prime}\right) \cong \boldsymbol{G}(\varphi)$, an isomorphism of algebraic groups. More precisely, there exists some $\Omega \in \operatorname{SL}(\mathcal{V})$ such that both $\mathfrak{g}\left(\varphi^{\prime}\right)=\Omega \mathfrak{g}(\varphi) \Omega^{-1}$ and $\boldsymbol{G}\left(\varphi^{\prime}\right)=\Omega \boldsymbol{G}(\varphi) \Omega^{-1}$; see [J, Sect. 1] for details. 
4.1. The map $A \mapsto A^{\dagger}$. In Subsection 3.1 we defined involutions $\theta^{\varepsilon}$ of $\mathfrak{G}$; and thus symmetric pairs $\left(\mathfrak{G}, \mathfrak{G}_{1}^{\varepsilon}\right)$. Here we want to consider two more involutions. For that, let again the field $\mathbb{K}$ be arbitrary. If $U \in M_{n}(\mathbb{K})$, we define $U^{\tau}=s U^{t} \boldsymbol{s}$; recall that the map $U \mapsto U^{\tau}$ is the skew transpose. A straightforward verification proves the following basic lemma.

Lemma 4.2. (i) For any $U_{1}, U_{2} \in M_{n}(\mathbb{K})$, we have $\left(U_{1} U_{2}\right)^{\tau}=U_{2}^{\tau} U_{1}^{\tau}$.

(ii) For a block-matrix $A$ we define $A^{\dagger}$ as follows:

$$
A^{\dagger}=A_{\varepsilon}^{\dagger}=\left(\begin{array}{cc}
T^{\tau} & \varepsilon Y^{\tau} \\
\varepsilon Z^{\tau} & X^{\tau}
\end{array}\right), \quad \text { if } A=\left(\begin{array}{cc}
X & Y \\
Z & T
\end{array}\right) .
$$

The map $A \mapsto A^{\dagger}$ is a $\mathbb{K}$-linear automorphism of $M_{2 n}(\mathbb{K})$, satisfying $A^{\dagger \dagger}=A$ and $(A B)^{\dagger}=B^{\dagger} A^{\dagger}$, for all $A, B \in M_{2 n}(\mathbb{K})$; i.e., this map is an involution on $M_{2 n}(\mathbb{K})$. For $A \in G L(2 n, \mathbb{K})$, we have $\left(A^{-1}\right)^{\dagger}=$ $\left(A^{\dagger}\right)^{-1}$.

(iii) For any $A \in M_{2 n}(\mathbb{K})$, we have $S_{\varepsilon}^{t} A^{t} S_{\varepsilon}=A^{\dagger}$.

Now define a Lie algebra

$$
\mathfrak{L}_{1}^{\varepsilon}=\left\{A \in \mathfrak{G} \mid S_{\varepsilon} A+A^{t} S_{\varepsilon}=0\right\}=\left\{A \in \mathfrak{G} \mid A^{\dagger}=-A\right\} ;
$$

we write just $\mathfrak{L}_{1}^{+}$and $\mathfrak{L}_{1}^{-}$. A block-matrix $A$, with $n$-by- $n$ blocks, is in $\mathfrak{L}_{1}^{\varepsilon}$ if and only if $A=\left(\begin{array}{cc}X & Y \\ Z & -X^{\tau}\end{array}\right)$, where $Y=-\varepsilon Y^{\tau}$ and $Z=-\varepsilon Z^{\tau}$. In other words, for $\varepsilon=1$ (resp. $\varepsilon=-1$ ) the matrices $Y$ and $Z$ are symmetric (resp. skew-symmetric) with respect to the skew diagonal. As the map

$$
\Theta=\Theta^{\varepsilon}: A \mapsto-A_{\varepsilon}^{\dagger}
$$

is an involutive automorphism of $\mathfrak{G}$, the pair $\left(\mathfrak{G}, \mathfrak{L}_{1}^{\varepsilon}\right)$ is symmetric. We have $\mathfrak{L}_{1}^{+}=\mathfrak{s o}(2 n, \mathbb{K})$ and $\mathfrak{L}_{1}^{-}=\mathfrak{s p}(2 n, \mathbb{K})$.

4.2. A comparison of $\theta^{\varepsilon}$ and $\Theta^{\varepsilon}$. By the above constructions we have Lie algebras $\mathfrak{G}_{1}^{\varepsilon}$ and $\mathfrak{L}_{1}^{\varepsilon}$. Next, for $G=\operatorname{SL}(2 n, \mathbb{K})$, we have the corresponding groups

$$
\begin{aligned}
G_{1}^{\varepsilon} & =G_{1}^{ \pm}=\left\{A \in G \mid A A_{\varepsilon}^{\sharp}=I\right\}, \\
L_{1}^{\varepsilon} & =L_{1}^{ \pm}=\left\{A \in G \mid A A_{\varepsilon}^{\dagger}=I\right\} ;
\end{aligned}
$$

for $G_{1}^{\varepsilon}$ see Subsection 3.3. Thus $G_{1}^{-}$and $L_{1}^{-}$(resp. $G_{1}^{+}$and $L_{1}^{+}$) are two different realizations of $\operatorname{Sp}(2 n, \mathbb{K})$ (resp. $\operatorname{SO}(2 n, \mathbb{K})$ ) within $G$. The following easy lemma states that these two pairs of algebraic structures are conjugate (we already know that for $\mathbb{K}$ algebraically closed they are; see the paragraph before Subsection 4.1). Therefore, by Lemma 4.1, in particular the normalizers $\mathcal{N}_{G}\left(G_{1}^{\varepsilon}\right)$ and $\mathcal{N}_{G}\left(L_{1}^{\varepsilon}\right)$ are conjugate as well. In other words, as we already said in the Introduction, Theorem 0.1 holds when we replace $G_{1}=G_{1}\left(\theta^{\varepsilon}\right)=G_{1}^{\varepsilon}$ there by $L_{1}^{\varepsilon}$.

LEMMA 4.3. We have the following: 
(i) $\mathfrak{L}_{1}^{\varepsilon} \cong \mathfrak{G}_{1}^{\varepsilon}$, conjugate Lie algebras.

(ii) $G_{1}\left(\omega_{ \pm}\right) \cong L_{1}^{ \pm}$, conjugate (abstract) groups.

Proof. (i) Let us show that there exists $\Omega$, $\operatorname{det} \Omega \in\{ \pm 1\}$, such that the map $\phi: \mathfrak{L}_{1}^{\varepsilon} \rightarrow \mathfrak{G}_{1}^{\varepsilon}, \phi(A)=\Omega A \Omega^{-1}$, is a Lie algebra isomorphism. For that purpose define $U=J_{\varepsilon}^{-1} S_{\varepsilon}=\left(\begin{array}{ll}s & 0 \\ 0 & s\end{array}\right)$. Then we clearly have $A^{\sharp}=U A^{\dagger} U^{-1}$, and therefore

$$
\phi(A)^{\sharp}+\phi(A)=\left(\Omega^{\sharp}\right)^{-1} U A^{\dagger} U^{-1} \Omega^{\sharp}+\Omega A \Omega^{-1} ;
$$

note that $U^{-1}=U$. If we can find such $\Omega$ that $U=\Omega^{\sharp} \Omega$, we are done. It remains to note that $\Omega=\left(\begin{array}{ll}s & 0 \\ 0 & I\end{array}\right)$ will do.

(ii) The map $\phi: L_{1}^{\varepsilon} \rightarrow G_{1}(\omega)$, defined in the same way as in (i), is an isomorphism of groups. To see this one just has to note that

$$
\phi(A)^{\sharp} \phi(A)=\left(\Omega^{\sharp}\right)^{-1}\left(A^{\sharp} U\right) A \Omega^{-1}=\left(\Omega^{\sharp}\right)^{-1} U A^{\dagger} A \Omega^{-1}=\left(\Omega^{\sharp}\right)^{-1} U \Omega^{-1}=I .
$$

Remark 4.4. Recall that the standard Borel subgroup $B$ of $G$ is the subgroup of $G$ consisting of the upper triangular matrices. For such $B$, define

$$
B_{1}=B_{1}^{\varepsilon}=B \cap L_{1}^{\varepsilon} .
$$

A well known fact is that then $B_{1}$ is a standard Borel subgroup of $L_{1}^{\varepsilon}$. This is one of the main reasons why the realization of $\operatorname{Sp}(2 n, \mathbb{K})(\operatorname{resp} . \mathrm{SO}(2 n, \mathbb{K}))$, via $\Theta^{\varepsilon}$, is interesting; cf. [Š6, Sect. 3]. Notice that there is no analogous result for the embedding of $G_{1}^{\varepsilon}$ into $G$.

4.3. A comparison of $\theta^{+}$and $\vartheta$. For $G=\operatorname{SL}(2 n, \mathbb{K})$, define a subgroup $H_{1} \leq G$ by

$$
H_{1}=\left\{A \in G \mid A A^{t}=I\right\}
$$

for $\mathbb{K}=\mathbb{R}$ or $\mathbb{C}$ we obtain the familiar realizations $H_{1}=\mathrm{SO}(2 n, \mathbb{R})$ or $\mathrm{SO}(2 n, \mathbb{C})$. Our next step is to compare the groups $H_{1}$ and $G_{1}^{+}$, where $G_{1}^{+}$is as in the previous subsection. Similarly, we treat the Lie algebras $\mathfrak{G}_{1}^{+}$and

$$
\mathfrak{H}_{1}=\left\{A \in \mathfrak{G} \mid A^{t}=-A\right\}
$$

note that $\mathfrak{H}_{1}=\mathfrak{G}^{\vartheta}$, for the involution

$$
\vartheta: A \mapsto-A^{t}
$$

of $\mathfrak{G}$. We begin with an interesting observation in the positive characteristic case; cf. the previous lemma, and see Remark 4.6 below.

LEMma 4.5. Let $\mathbb{K}$ be a field of characteristic p, and suppose that either $p \equiv 1(\bmod 4)$, or $p \equiv 3(\bmod 4)$ and $\mathbb{K}$ contains $\mathbb{F}_{p^{2}}$ as a subfield. Then we have the following:

(i) $G_{1}^{+} \cong H_{1}$, conjugate groups.

(ii) $\mathfrak{G}_{1}^{+} \cong \mathfrak{H}_{1}$, conjugate Lie algebras. 
Proof. (i) Let us show, again, that there exists some $\Omega$ such that $\phi$ : $H_{1} \rightarrow G_{1}^{+}, \phi(A)=\Omega A \Omega^{-1}$, is an isomorphism. Since

$$
\phi(A)^{\sharp} \phi(A)=\left(\Omega^{-1}\right)^{\sharp} J_{+} A^{t} J_{+} \Omega^{\sharp} \Omega A \Omega^{-1},
$$

it is obvious that we would have what we want provided that $\Omega^{\sharp} \Omega=J_{+}$. Write now $\Omega=\left(\begin{array}{cc}X & Y \\ Z & T\end{array}\right)$. Thus $\Omega^{\sharp} \Omega=J_{+}$is equivalent to the following:

$$
T^{t} X+Y^{t} Z=0, \quad T^{t} Y+Y^{t} T=I=Z^{t} X+X^{t} Z .
$$

Consider the case $n=1$, i.e., $\Omega=\left(\begin{array}{ll}x & y \\ z & t\end{array}\right)$ with $x, y, z, t \in \mathbb{K}$. By the assumption on $\mathbb{K}$, we can find some nonzero $x, y$ satisfying $x^{2}+y^{2}=0$. Then put $z=1 / 2 x$ and $t=1 / 2 y$. Now it follows that

$$
\Omega=\left(\begin{array}{ll}
x I & y I \\
z I & t I
\end{array}\right)
$$

will work for any $n$; note that $\operatorname{det} \Omega=(x t-y z)^{n}$, and so $\Omega \in \mathrm{GL}(2 n, \mathbb{K})$, but $\Omega \notin G$ for $n$ not divisible by 4 .

(ii) The map $\phi: \mathfrak{H}_{1} \rightarrow \mathfrak{G}_{1}^{+}$, defined as in (i), is an isomorphism of Lie algebras.

REMARK 4.6. Since $\mathfrak{H}_{1}$ consists of all block-matrices $\left(\begin{array}{cc}X & Y \\ -Y^{t} & T\end{array}\right)$ satisfying $X+X^{t}=T+T^{t}=0$, in particular for $n=1$ we have $\mathfrak{H}_{1}$ to be the set of all $\left(\begin{array}{cc}0 & y \\ -y & 0\end{array}\right), y \in \mathbb{K}$. Also, for $n=1, \mathfrak{G}_{1}^{+}$equals the set of all $\left(\begin{array}{cc}x & 0 \\ 0 & -x\end{array}\right), x \in \mathbb{K}$. Thus, for arbitrary $\mathbb{K}$, we have $\mathfrak{H}_{1} \cong \mathfrak{G}_{1}^{+}$, in the obvious way.

On the other hand the situation with groups is different. To see this let, again, $n=1$. Then for $\mathbb{K}=\mathbb{F}_{3}$ we have $H_{1} \cong \mathbb{Z} / 4 \mathbb{Z}$ and $G_{1}^{+} \cong \mathbb{Z} / 2 \mathbb{Z}$. Also, for $\mathbb{K}=\mathbb{R}$, the subgroups $G_{1}^{+}$and $H_{1}$ of $\operatorname{SL}(2, \mathbb{R})$ are not isomorphic as well. Of course, these are just special cases of a more general situation concerning the orthogonal groups; cf. [Ca, Sect. 1.19].

ExAmple 4.7. Let $G$ and $H_{1}$ be as above. As in Theorem 3.7, we have that if $M \in \mathcal{N}_{G}\left(H_{1}\right)$, then $M^{t} M \in \mathcal{C}_{G}\left(H_{1}\right)$.

Let $n=1$ and $H_{1} \leq G=\mathrm{SL}(2, \mathbb{K})$, i.e.,

$$
H_{1}=\left\{\left(\begin{array}{cc}
a & b \\
-b & a
\end{array}\right) \mid a, b \in \mathbb{K} \text { satisfying } a^{2}+b^{2}=1\right\} .
$$

It is easy to see that for the centralizer of $H_{1}$ in $G$ we have $\mathcal{C}_{G}\left(H_{1}\right)=H_{1}$. We want to describe the normalizer of $H_{1}$ in $G$. So let now $M=\left(\begin{array}{cc}x & y \\ z & t\end{array}\right) \in \mathcal{N}_{G}\left(H_{1}\right)$. Hence, $M^{t} M \in H_{1}$; i.e., $x^{2}+z^{2}=a=y^{2}+t^{2}$ and $-b=x y+z t=b$, for $a, b$ satisfying $a^{2}+b^{2}=1$. Clearly, for $b \neq 0, M$ does not exist. So it follows that $a= \pm 1$. An easy calculation shows that for $a=1$ we have $M \in H_{1}$. Also, for $a=-1$, we deduce that $M$ is of the form

$$
M=M(x ; y)=\left(\begin{array}{cc}
x & y \\
y & -x
\end{array}\right), \quad \text { where } x^{2}+y^{2}+1=0 .
$$


Thus we have the normalizer of $H_{1}$ in $G$ decomposed as a (disjoint) union $\mathcal{N}_{G}\left(H_{1}\right)=H_{1} \cup \Phi$, where $\Phi$ is the set of all matrices $M(x ; y)$. If $\Phi$ is nonempty, fix an arbitrary matrix $\varpi \in \Phi$. Then note that the map $H_{1} \ni h \mapsto \varpi h \in \Phi$ is bijective; thus, $\Phi=\varpi H_{1}$. Also define a map

$$
\sigma: \mathcal{N}_{G}\left(H_{1}\right) \rightarrow \mathbb{Z} / 2 \mathbb{Z}=\{\overline{0}, \overline{1}\}, \quad \sigma_{\mid H_{1}}=\overline{0}, \quad \sigma_{\mid \Phi}=\overline{1}
$$

Using the fact that $h \varpi=\varpi h^{t}$, for every $h \in H_{1}$, it is obvious that $\sigma$ is an epimorphism, with the kernel $\operatorname{Ker} \sigma=H_{1}$. The above considerations can be summarized as follows.

ClAIM. Supposing that the equation $x^{2}+y^{2}+1=0$ has a solution in $\mathbb{K}$, we have a short exact sequence of groups

$$
1 \rightarrow H_{1} \hookrightarrow \mathcal{N}_{G}\left(H_{1}\right) \stackrel{\sigma}{\rightarrow} \mathbb{Z} / 2 \mathbb{Z} \rightarrow 1 .
$$

Otherwise, we have $\mathcal{N}_{G}\left(H_{1}\right)=H_{1}$.

ACKNOWLEDGEMENTS.

The author is very grateful to the referee for a thorough reading of the manuscript. The referee's criticism and suggestions helped to improve the organization of the paper in a significant way.

\section{REFERENCES}

[A] E. Artin, Geometric Algebra, Interscience, New York, 1957.

[Bo] A. Borel, Linear Algebraic groups. Second Enlarged Edition, Graduate Texts in Math., Vol. 126, Springer-Verlag, New York, 1991.

[B1] N. Bourbaki, Lie groups and Lie algebras, Chapters 1-3, Springer-Verlag, Berlin, 1989.

[B2] N. Bourbaki, Groupes et Algèbres de Lie, Chapitres VII, VIII, Hermann, Paris, 1975.

[BK] R. Brylinski and B. Kostant, Nilpotent orbits, normality, and Hamiltonian group actions, J. Amer. Math. Soc. 7 (1994), 269-298.

[Ca] R. Carter, Finite groups of Lie type. Conjugacy classes and complex characters, Wiley-Interscience, Chichester, 1993.

[Ch] C. Chevalley, Sur certains groupes simples, Tôhoku Math. J (2) 7 (1955), 14-66.

[D] J. Dixmier, Enveloping Algebras, Graduate Studies in Mathematics 11, Amer. Math. Soc., 1996.

[Dy1] E. B. Dynkin, Semisimple subalgbras of semisimple Lie algebras, Amer. Math. Soc. Translations (2) 6 (1957), 111-244.

[Dy2] E. B. Dynkin, Maximal subgroups of the classical groups, Amer. Math. Soc. Translations (2) 6 (1957), 245-378.

[GW] R. Goodman and N. R. Wallach, Representations and Invariants of the Classical Groups, Encyclopedia of Mathematics and its Applications, Vol. 68, Cambridge Univ. Press, Cambridge, 1998.

[HTW] R. Howe, E.-C. Tan and J. F. Willenbring, Stable branching rules for classical symmetric pairs, Trans. Amer. Math. Soc. 357 (2005), 1601-1626.

[Hu] J. E. Humphreys, Conjugacy Classes in Semisimple Algebraic Groups, Mathematical Surveys and Monographs, Vol. 43, Amer. Math. Soc., Providence, 1995.

[J] J. C. Jantzen, Nilpotent orbits in representation theory, Lie Theory, Progr. Math., Vol. 228, Birkhäuser, Boston (2004), pp. 1-211. 
[Kn] A. W. Knapp, Geometric interpretations of two branching theorems of D. E. Littlewood, J. Algebra 270 (2003), 728-754.

[Ko1] T. Kobayashi, Discrete decomposability of the restriction of $A_{\mathfrak{q}}(\lambda)$ with respect to reductive subgroups III. Restriction of Harish-Chandra modules and associated varieties, Invent. Math. 131 (1998), 229-256.

[Ko2] T. Kobayashi, Discretely decomposable restrictions of unitary representations of reductive Lie groups-examples and conjectures, Analysis on homogeneous spaces and representation theory of Lie groups, Okayama-Kyoto (1997), 99-127, Adv. Stud. Pure Math. 26, Math. Soc. Japan, Tokyo, 2000.

[Kob] N. Koblitz, A course in number theory and cryptography, 2nd ed., Graduate Texts in Math., Vol. 114, Springer-Verlag, Berlin, 1994.

[Ks] B. Kostant, A branching law for subgroups fixed by an involution and a noncompact analogue of the Borel-Weil theorem, Noncommutative harmonic analysis, Progress in Math., vol. 220, Birkhäuser Boston, Boston, 2004, pp 291-353.

[LS] T. Levasseur and S. P. Smith, Primitive ideals and nilpotent orbits in type $G_{2}, \mathrm{~J}$. Algebra 114 (1988), 81-105.

[R] R. W. Richardson, Jr., Conjugacy classes in Lie algebras and algebraic groups, Ann. of Math. (2) 86 (1967), 1-15.

[S] G. M. Seitz, The maximal subgroups of classical algrebraic groups, Mem. Amer. Math. Soc., 67 No. 365 (1987).

[ك̌1] B. Širola, On pairs of complex Lie groups and generalized global Cartan decomposition, Grazer Math. Ber. 348 (2005), 79-90.

[Š2] B. Širola, A generalized global Cartan decomposition: a basic example, Comm. Algebra 34 (2006), 3267-3279.

[Š3] B. Širola, Pairs of semisimple Lie algebras and their maximal reductive subalgebras, Algebr. Represent. Theory 11 (2008), 233-250.

[Š4] B. Širola, Pairs of Lie algebras and their self-normalizing reductive subalgebras, J. Lie Theory 19 (2009), 735-766.

[ک̌5] B. Širola, Normalizers and self-normalizing subgroups II, Cent. Eur. J. Math. 9 (2011), 1317-1332.

[ک̌6] B. Širola, On centralizers and normalizers for groups, preprint.

[SS] T. A. Springer and R. Steinberg, Conjugacy classes, Seminar on Algebraic Groups and Related Finite Groups (A. Borel et al. eds.), Lecture Notes in Math., Vol. 131, Springer-Verlag, Berlin, 1970, pp. 167-266.

[V] D. A. Vogan, Jr., The unitary dual of $G_{2}$, Invent. Math. 116 (1994), 677-791.

[Wa] A. Wagner, On the clssification of the classical groups, Math. Zeit. 97 (1967), $66-76$.

[Wl] G. E. Wall, On the conjugacy classes in the unitary, symplectic and orthogonal groups, J. Austral. Math. Soc. 3 (1963), 1-62.

B. Širola

Department of Mathematics

University of Zagreb

Bijenička 30, 10000 Zagreb

Croatia

E-mail: sirola@math.hr

Received: 3.8.2010.

Revised: 7.1.2011. \& 13.7.2011. 